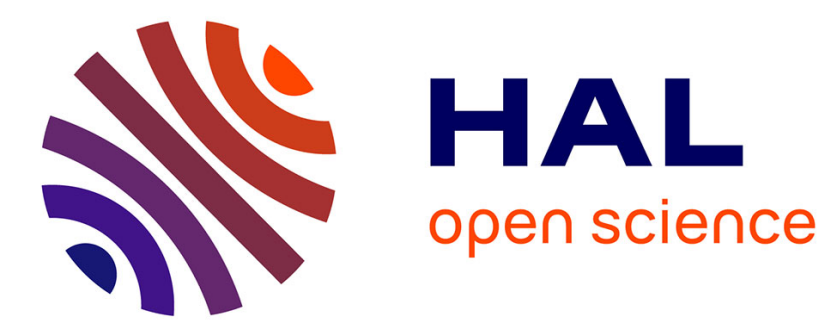

\title{
Dispersive Radon transform
}

Kailiang Xu, Pascal Laugier, Jean-Gabriel Minonzio

\section{To cite this version:}

Kailiang Xu, Pascal Laugier, Jean-Gabriel Minonzio. Dispersive Radon transform. Journal of the Acoustical Society of America, 2018, 143 (5), pp.2729-2743. 10.1121/1.5036726 . hal-01971783

\section{HAL Id: hal-01971783 https://hal.sorbonne-universite.fr/hal-01971783}

Submitted on 7 Jan 2019

HAL is a multi-disciplinary open access archive for the deposit and dissemination of scientific research documents, whether they are published or not. The documents may come from teaching and research institutions in France or abroad, or from public or private research centers.
L'archive ouverte pluridisciplinaire HAL, est destinée au dépôt et à la diffusion de documents scientifiques de niveau recherche, publiés ou non, émanant des établissements d'enseignement et de recherche français ou étrangers, des laboratoires publics ou privés. 


\title{
Dispersive Radon transform
}

\author{
Kailiang $\mathrm{Xu},{ }^{\mathrm{a})}$ Pascal Laugier, and Jean-Gabriel Minonzio \\ Sorbonne Universités, UPMC Univ Paris 06, CNRS, INSERM, Laboratoire d'Imagerie Biomédicale (LIB), \\ 15 rue de l'école de médecine, 75006, Paris, France
}

\begin{abstract}
Dispersion results in the spreading and overlapping of the wave-packets, which often limits the capability of signal interpretation; on the other hand, such a phenomenon can also be used for structure or media evaluation. In this study, the authors propose an original dispersive Radon transform (DRT), which is formulated as integration transform along a set of dispersion curves. Multichannel dispersive signals of each individual mode can be concentrated to a well localized region in the DRT domain. The proposed DRT establishes the sparse projection of the dispersive components and provides an efficient solution for mode separation, noise filtering, and missing data reconstruction. Particularly the DRT method allows projecting the temporal signals of dispersive waves on the space of parameters of interest, which can be used to solve the inverse problem for waveguide or media property estimation. The least-square procedure and sparse scheme of the DRT are introduced. A high-resolution DRT is designed based on an iterative reweighting inversion scheme, which resembles the infinite-aperture velocity gather. The proposed method is applied by analyzing ultrasonic guided waves in plate-like structures and in a human radius specimen. The results suggest that the DRT method can significantly enhance the interpretation of dispersive signals.
\end{abstract}

\section{INTRODUCTION}

Dispersion is a phenomenon in which the properties of a propagating wave-packet depend on frequency. In the signal processing community, considerable efforts have been attracted surrounding different applications, such as radar, ${ }^{1-3}$ communication, ${ }^{4,5}$ non-destructive evaluation, ${ }^{6-8}$ seismology, ${ }^{9-12}$ underwater acoustics, ${ }^{13,14}$ and some biomedical applications. ${ }^{15,16}$ Depending on its causes, it can be generally classified as media dispersion and waveguide dispersion. Both can bring desirable or undesirable effects. From the signal processing point of view, for undesirable case ${ }^{1-5,17}$ the effects of dispersion cause the wave-packets to spread spatially and temporally during their propagation, which limits the capability of signal interpretation, so that one of the main objectives is to remove the effect of dispersion, such as dispersion compensation or wave-packet separation; on the other hand, the dispersion effect can actually be measured, ${ }^{6-16}$ which denotes its transfer function can be quantitatively determined and further used for media characterization and waveguide evaluation. Consequently, the major challenges for dispersive signal processing include dispersion suppression and dispersion based media or structure evaluation.

Since the 1980 s, the classical time-frequency representation (TFR) method ${ }^{18}$ for dispersive signals has become popular in the signal processing community. The TFR method has been applied to analyze the wideband electromagnetic scattering, ${ }^{19}$ seismic waves, ${ }^{9}$ ultrasonic guided waves, ${ }^{7}$ and radar signal, ${ }^{1}$ etc. Many improved TFR-based

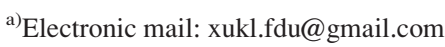

strategies of dispersion curves extraction and mode separation have been proposed in different fields, such as the group delay shift covariant quadratic $\mathrm{TFR}^{20}{ }^{20}$ power-class TFR, ${ }^{21}$ dispersion-based TFR, ${ }^{22}$ Chirplet transform, ${ }^{23}$ and matched TFR representation, ${ }^{24}$ etc. These methods are mainly developed by decomposing the signals into TFR atoms whose group delays are modulated in frequency corresponding to the wave dispersion. One of the major advantages of the TFR-based dispersion analysis is that the single-channel, i.e., one emitter and one receiver or backscatter setup, is only required for signal measurement. However, such a single-channel measurement also limits the recorded information, making it challenging to extract all dispersion characteristics, especially for low-amplitude modes.

The approaches combining multichannel recording with multichannel processing have been employed to improve mode separation and noise filtering. ${ }^{10,12,25,26}$ Among these, the most straightforward one is the two-dimensional Fourier transform (2D-FT), in which the recorded data in the timedistance domain is projected into the frequency-wavenumber space with the energy locus corresponding to the dispersion curves. $^{10,27}$ However, the limited number of positions to record the multichannel signals (i.e., the limited aperture problem) results in a limited resolution of the representation of the dispersion curves obtained using the classical 2D-FT.

Considering the sparsity of the dispersion curves in the frequency-wavenumber domain, the compressed sensing method has thus been applied for high-resolution recovery of the dispersion curves of Lamb modes. ${ }^{28,29}$ Courtois and Bonnel have introduced the compressed sensing method for wideband wavenumber tracking in dispersive shallow water. ${ }^{30}$ Such a method has been further developed to reduce the required number of sensors for an accurate wideband 
wavenumber separation. ${ }^{31}$ Recently, our team proposed a sparse singular value decomposition (S-SVD) method, ${ }^{8}$ which combines the sparse wavenumber regularization strategy and SVD-based noise filtering, ${ }^{26}$ to achieve a highresolution extraction of the dispersion curves of ultrasonic guided waves in attenuated medium, such as transversely isotropic composite plate and human long cortical bone.

An alternative solution to the classical Fourier dictionary based method is Radon transform (RT), also known as slant stack transform, an integration transform introduced by Johan Radon in 1917. ${ }^{32,33}$ The RT establishes an efficient solution for the forward and inverse transformation between a line event in Cartesian coordinate space and a point in polar coordinate space. Based on this property, the RT has been introduced for seismic signal processing, especially for ray event identification and velocity determination. ${ }^{33}$ Ambrozinski et $a l .{ }^{34}$ applied the RT for ultrasonic guided waves signal processing, and it was demonstrated that RT provides the similar results of wave velocity and mode content as the 2D-FT method. To overcome the limitation of a finite aperture of the spatial discretization, Thorson and Claerbout ${ }^{35}$ proposed the least-squares stochastic inversion, leading to the high-resolution RT method. Luo et al. ${ }^{36}$ applied the high-resolution RT method for sparse dispersive energy imaging of the Rayleigh waves. The high-resolution RT method applied to ultrasonic guided waves in cortical bone has been reported to be able to enhance the resolution of the extracted dispersion curves in comparison to the 2DFT method. ${ }^{37}$ However, in our recent report, ${ }^{38}$ we found that the high-resolution RT is not well adapted to concentrate the dispersion trajectories of low-amplitude modes when no clear linear or parabolic ray events can be observed in the wideband multimode dispersive signal. Furthermore, the dispersion effect causes the inefficiency of the sparse penalty, which denotes that the high-resolution RT is not suitable for dispersive signal processing.

The ultimate objective for dispersion curves extraction is for the media or structure evaluation. The estimated dispersion function is often used to fit a forward model with some unknown parameters, e.g., structure thickness, density, elasticity coefficients, and electromagnetic coefficients, etc. After solving an inverse problem, those parameters can be inferred by minimizing the discrepancy between the extracted dispersion characteristics and the forward model prediction. As aforementioned, currently numerous methods have been available for processing dispersive signals, but to the best of the authors knowledge, most of them still focus on characterizing the dispersion function rather than directly solving the inverse problem. Such a gap brings many challenges for dispersion-based applications, such as ultrasonic guided wave based non-destructive evaluation. On the contrary, there is currently a large literature discussing many aspects of an inverse-problem-based model fitting strategy, such as the so-called matched field processing for source localization, ${ }^{39,40}$ estimation of deep ocean sound-speed profiles, ${ }^{41}$ and recent defect imaging, ${ }^{42}$ etc.

This study illustrates that the strategy of inverse-problem-based model fitting can be introduced to overcome the limitation of the classical RT transform for wideband dispersive signal processing, leading to a novel dispersive Radon transform (DRT) method. In addition, being designed with a dispersive tiling, the DRT transform allows a considerable reduction of dispersive energy smearing and further enables to directly solve the inverse problem for dispersionbased media or structure evaluation. Instead of only fitting the measured data, a sparse penalization strategy with the assumption of data missing is presented to improve the noise-filtering and to enhance the resolution in the DRT domain.

This paper is organized as follows. The forward and inverse dispersive Radon transform, i.e., DRT and IDRT, are formulated with a discrete realization of the corresponding algorithm. The least-square solution of the inverse scheme is implemented. Furthermore, the sparse penalization technique is introduced. The performance of the DRT method is investigated with the ultrasonic guided waves in the plate-like structure, including the synthetic data, experimental signals measured from a transversely isotropic plate, and a human radius (can be seen as a shell-like waveguide with transversely isotropic elasticity). The advantages of the proposed DRT method are finally discussed.

\section{THEORY AND METHODS}

\section{A. Guided waves dispersion}

Multimodal ultrasonic guided waves are presented in this study as examples for the DRT method. Please refer to the references in specific fields with respect to the theoretical model of other dispersive signals, such as guided waves in shallow water, ${ }^{43}$ Rayleigh waves in seismology, ${ }^{36}$ or material dispersion, such as soil. ${ }^{44}$

According to the Rayleigh-Lamb equation, guided waves in a plate structure, called Lamb modes, are classified as symmetric (S) and antisymmetric (A) modes, briefly named as $S n$ and $A n$ modes $(n=0,1,2, \ldots) .{ }^{45}$ With the known values of material parameters, dispersion curves can be expressed as wavenumber $k$ versus angular-frequency $\omega$. Let $g(x, t)$ represent a distance-time matrix measured using a single emitter and a multiple receiver array. The propagation function of a certain guided mode can be described as integration along a given dispersion curve

$$
g(x, t)=\int_{-\infty}^{+\infty} F(\omega) e^{j(k(\omega) x-\omega t)} d \omega
$$

where $g(x, t)$ is the series of temporal signals recorded at a series of space positions $x . F(\omega)$ is the spectrum of the excitation signal with $\omega=2 \pi f$. For generating the synthetic signals, we used $F(\omega)=0.5 *\left[1-\cos \left(\omega / \omega_{0}\right)\right], 0<\omega<\omega_{0}$, and $\omega_{0}=0.8 \mathrm{MHz}$ with a $-6 \mathrm{~dB}$ bandwidth of $0.4 \mathrm{MHz}$. With the known dispersion curve $k(\omega)$, the spectrum of the dispersive signal can thus be computed by multiplying a phase adjustment term $e^{j k(\omega) x}$, and then the temporal waveforms of the multichannel synthetic signals can be obtained by inverse Fourier transform (FT). ${ }^{17}$ 


\section{B. Definition of the fundamental DRT}

The FT pair between the spatial and wavenumber domains is given by

$$
\begin{aligned}
& U(k, \omega)=\int_{x} G(x, \omega) e^{j k x} d x, \\
& G(x, \omega)=\int_{k} U(k, \omega) e^{-j k x} d k .
\end{aligned}
$$

As shown in Fig. 1(a), the dispersion curve of a given mode cannot match the $(f, k)$ basis tiling obtained using the FT. The energy of a set of distance-shifting dispersive signals will spread over many basis coefficients.

$\mathrm{RT}$ is defined by an integral operator along a given line of $t=\tau+p x$, where $\tau$ and $p$ denote the intercept time and slope or phase slowness in seismic application and $p=k / \omega$. Thus, RT is also called $\tau-p$ transform. In the frequency domain, the pair of RT is given by ${ }^{46}$

$$
\begin{aligned}
R(p, \omega) & =\int_{x} G(x, \omega) e^{j \omega p x} d x, \\
G(x, \omega) & =\int_{p} \omega R(p, \omega) e^{-j \omega p x} d p .
\end{aligned}
$$

In Eq. (3a), array signals in $(x, \omega)$ space are projected into the $(p, \omega)$ domain. The transformation in Eq. (3b) maps the $(p, \omega)$ space into the $(x, \omega)$ domain. Taking the FT on $\omega$, Eq. (3) yields a RT pair between $(p, \tau)$ and $(x, t)$. In the seismology field, Eq. (3a) is usually called back-projection, as the information propagated from the transfer function [velocity model in $(p, \omega)$ space] to signal is projected backward into the slowness-frequency domain. ${ }^{46}$ The linear $\tau-p$ transform provides an efficient way for projecting each ray event in the $(x, t)$ domain to the $(\tau, p)$ fields as a concentrated region. Taking into account the slowness $p=k / \omega$, the linear Radon tiling can trace the event with wave-packets propagating at a constant velocity [see Fig. 1(b)].

When no clear linear or parabolic ray event can be observed in the dispersive signals, e.g., wideband guided waves, ${ }^{34,38}$ the classical linear or quadratic RT cannot achieve a sparse concentration in the $(\tau, p)$ field. In other words, the dispersive signal cannot fit the Radon tiling. We seek a nonlinear Radon projection with the capability of a dispersive velocity gather. DRT operators are thus defined by an integral transform along a family of dispersion curves $k(\omega, s)$. In the frequency domain, the pair of back- and forward-projection DRT operators is given by

$$
\begin{aligned}
& W(s, \omega)=\int_{x} G(x, \omega) e^{j k(s, \omega) x} d x, \\
& \hat{G}(x, \omega)=\int_{s} W(s, \omega) e^{-j k(s, \omega) x} d s,
\end{aligned}
$$

where $k(s, \omega)$ is the dispersion curve of a given mode and $s$ could be a specific parameter of interest, for instance, thickness, stiffness coefficient, or density. Compared to Eq. (3), Eq. (4) extends the classical RT to a more general case with dispersion function.

Substituting Eq. (4a) into Eq. (4b), we obtain

$$
\hat{G}(x, \omega)=\int_{x^{\prime}} G\left(x^{\prime}, \omega\right) \int_{s} e^{-j k(s, \omega)\left(x-x^{\prime}\right)} d s d x^{\prime}
$$

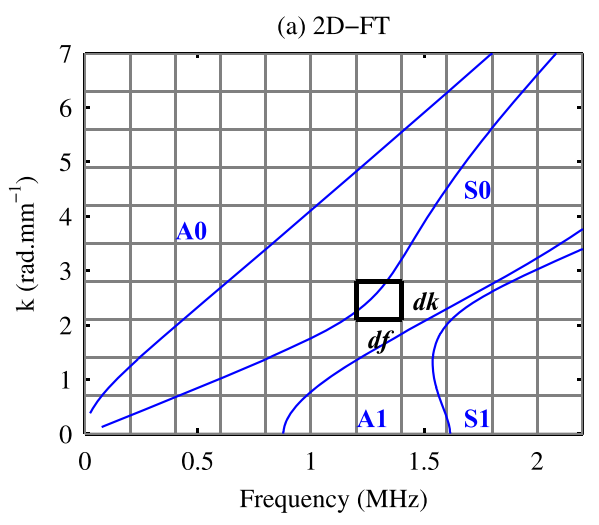

(c) Dispersive Radon transform (S0)

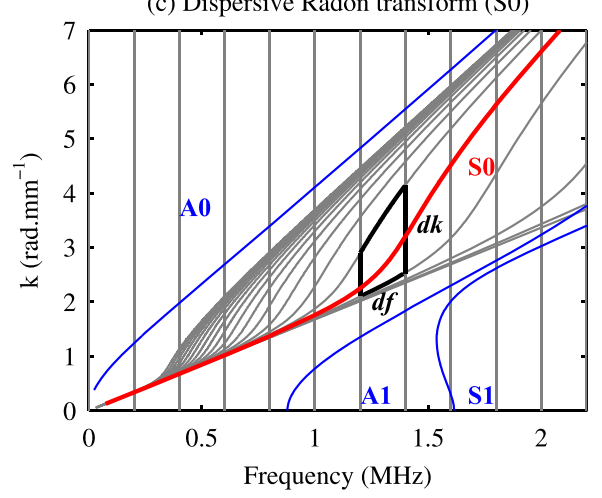

(b) Radon transform

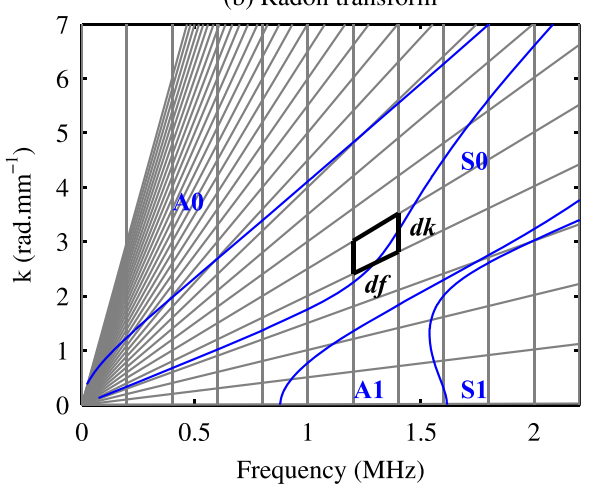

(d) Dispersive Radon transform (A1)

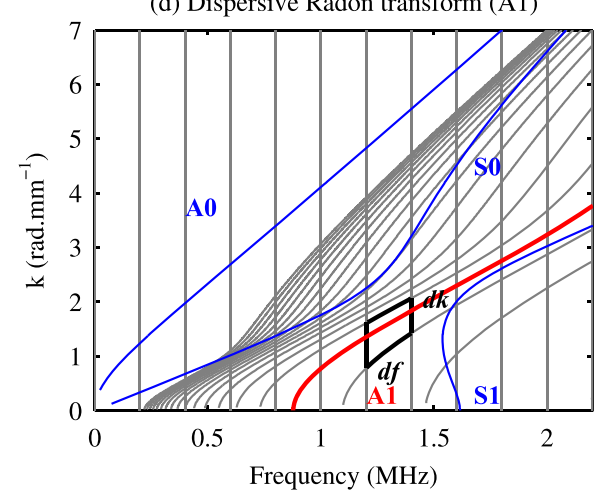

FIG. 1. (Color online) Tiling of the $(f, k)$ plane, (a) 2D-FT, (b) RT with the slowness range $0.01<p$ $<15 \mathrm{rad}(\mathrm{mm} \mathrm{MHz})^{-1}$, DRT using the dispersion curves of S0 mode (c) and A1 mode (d), with the thickness range $0.2<s<4 \mathrm{~mm}$. The dispersion curves of the four fundamental Lamb modes calculated using the cortical bone parameters listed in Table I are plotted as solid lines. The red lines in (c) and (d) are dispersion curves of two selected modes S0 and A1, being used to obtain two corresponding DRT tiling. 
It can be rewritten as follows:

$$
\begin{aligned}
& \hat{G}(x, \omega)=G(x, \omega) * \varphi(x, \omega), \\
& \varphi(x, \omega)=\int_{s} e^{-j k(s, \omega) x} d s,
\end{aligned}
$$

where $*$ denotes convolution. It illustrates that $G(x, \omega)$ could be recovered after solving a deconvolution. Without a closed form expression, direct deconvolution of $\varphi(x, \omega)$ can be difficult. To solve this problem, a high resolution DRT method is thus applied (see Secs. II D and II E).

As examples, in Figs. 1(c) and 1(d) the nonlinear tilings of the $(f, k)$ plane using the DRT basis of S0 and A1 modes are presented, respectively, and the transform is shown with $s$ denoting thickness. It can be seen that different from the classical 2D-FT and RT, the DRT $(f, k)$ atoms closely follow a set of dispersion curves with thickness varying. The dispersive trajectory of a certain mode can thus be tracked as a concentrated region in the DRT domain. This property provides the advantage of the dispersive velocity gather and straightforwardly integrates the dispersion signal processing and the parameter estimation.

\section{Discrete DRT}

Instead of the exact inversion of RT defined in Eq. (3), a discrete analog expression has been widely accepted in a multitude of applications. ${ }^{33,46}$ Please refer to Ref. 33 for a well-written and complete description of the discrete Radon transform algorithm. For each frequency point, the analog transform matrices are

$$
\begin{aligned}
& R=M^{H} G, \\
& \tilde{G}=M R \\
& M=\left[\begin{array}{ccc}
e^{-j \omega p_{1} x_{1}} & \cdots & e^{-j \omega p_{N_{p}} x_{1}} \\
\vdots & \ddots & \vdots \\
e^{-j \omega p_{1} x_{N_{x}}} & \cdots & e^{-j \omega p_{N_{p}} x_{N_{x}}}
\end{array}\right]_{N_{x} \times N_{p}},
\end{aligned}
$$

where $N_{p}$ and $N_{x}$ are the number of sampling points of the slowness and distance, respectively, and $M^{H}$ is the complexconjugate transpose of $M . G$ and $R$ designate the array signals in the $(x, \omega)$ and $(p, \omega)$ domains, respectively. Equation (7) provides a convenient implementation of the RT pair using the algebraic reconstruction.

Similarly, for each frequency point, the analog discrete DRT transform matrices are given by

$$
\begin{aligned}
W & =L^{H} G \\
\hat{G} & =L W \\
L & =\left[\begin{array}{ccc}
e^{-j k\left(s_{1}, \omega\right) x_{1}} & \cdots & e^{-j k\left(s_{N_{s}}, \omega\right) x_{1}} \\
\vdots & \ddots & \vdots \\
e^{-j k\left(s_{1}, \omega\right) x_{N_{X}}} & \cdots & e^{-j k\left(s_{N_{s}, \omega}\right) x_{N_{X}}}
\end{array}\right]_{N_{x} \times N_{s}}
\end{aligned}
$$

Equation (8) forms an adjoint pair. $L^{H}$ is the complexconjugate transpose of $L$. Following the classical definition of RT in seismology field, $L^{H}$ and $L$ are also named as backprojection operator and forward-projection operator, respectively. After the inverse FT from frequency to time, the $(s, \omega)$ domain signals can be projected back to the $(s, \tau)$ domain, where $\tau$ defines the arriving time of the corresponding dispersive wave-packet. For abbreviation, the DRT is hereafter referred to as the $\tau-s$ transform. Since $L$ is nonunitary, $L^{H}$ and $L$ do not constitute an inverse pair. When additive noise is present, such a formulation cannot permit to model the signal completely, and the noise is mapped between two domains. ${ }^{46}$ A least-squares strategy can be introduced to obtain the least-squares DRT solution.

\section{Least-squares DRT}

A penalized least-squares solution to Eq. (8) can be obtained by minimizing the following cost function:

$$
J=\|G-L W\|_{2}^{2}+\mu Q(W),
$$

where the Lagrange multiplier $\mu$, also referred to as the regularization hyperpameter, ${ }^{46}$ determines the trade-off between the misfit term $\|G-L W\|_{2}^{2}$ and the penalty term $Q(W)$ in the $(s, \omega)$ domain.

A typical implementation of the penalty term is to use the quadratic $l_{2}$-norm, i.e., $Q(W)=\|W\|_{2}^{2}$, so that the leastsquares solution can be analytically obtained as

$$
\hat{W}=\left(L^{H} L+\mu I\right)^{-1} L^{H} G .
$$

In least-square estimation, no tacit assumption about the missing data is involved and only the existing data needs to be best-fit by the model. ${ }^{35}$ However, in practice we can only measure the signals over a limited range of position, which is the so-called limited aperture problem. In particular, when measuring guided wave signals, it results in a much lower resolution in the wavenumber axis, which can further decrease the resolution in the $(\tau, s)$ domain. Better strategy with the assumption of data missing is required.

\section{E. High-resolution DRT}

Enlightened by Burg, ${ }^{47}$ Thorson and Claerbout ${ }^{35}$ designed a stochastic inversion strategy to solve the finite aperture challenge, which finally leads to the high-resolution RT solution. The basic idea is to overcome the blurring spectral estimation by forcing the solution to be locally focused in a model space, so that the physical model fitting can be achieved with the best possible resolution. Therefore, mapping the data from such a sparse model-space to the arraysignal space not only selectively enhances the dispersive signals but also extrapolates the infinite-far offset, which can resemble the infinite-aperture velocity gather. A discussion can be found in Sec. IV A 2.

A common approach for obtaining a sparse transform is to choose a $l_{1}$-norm for the model and a $l_{2}$-norm for the data misfit, which is a mixed norm problem and can be solved by using an iteratively re-weighted least squares algorithm. ${ }^{48}$ 
Considering the sparsity of a physical model, i.e., sparsity of the dispersion curve, two typical non-quadratic penalty terms, i.e., $l_{1}$-norm $Q(W)=W_{1}$ and Cauchy norm, are usually adopted. The Cauchy-norm penalty term is defined as ${ }^{49}$

$$
Q(W)=\sum_{i=1}^{N_{p}} \ln \left[1+W\left(s_{i}, \omega\right) / \varepsilon^{2}\right] .
$$

According to the discrepancy principle, a proper $\varepsilon^{2}$ value of the Cauchy distribution should be selected to ensure that the misfit matches the power of noise. ${ }^{50}$ Since there is no analytical solution for the non-quadratic regularization, an iterative reweighting scheme ${ }^{50}$ is thus applied as follows:

(1) Initialization with the least-squares solution $\hat{W}^{(0)}$, at each frequency $\omega_{i}$, according to Eq. (10).

(2) Computing the reweighting matrix $Q^{(n)}$ of the $n$th iteration, using the $l_{1}$-norm or Cauchy norm. $Q^{(n)}$ is a diagonal matrix based on current $\hat{W}^{(n)}$. The diagonal entries of $Q^{(n)}$ are obtained by $\left(\left|G\left(x, \omega_{i}\right)\right|+b\right)^{-1}$ and $\left(\left|G\left(x, \omega_{i}\right)\right|^{2}+b\right)^{-1}$ for $l_{1}$-norm and Cauchy norm penalty, respectively. The hyperparameter $b$ ensures the continuity of the penalty function gradient and enforces the degree of sparsity. ${ }^{51}$

(3) Iteratively updating the estimation as follows:

$$
\hat{W}^{(n+1)}=\left[L^{H} L+\mu Q^{(n)}\right]^{-1} L^{H} G .
$$

(4) Convergence for the $n$th iteration, the procedure is stopped when the cost function converges according to

$$
\Delta J_{n}=\frac{\left|J^{(n+1)}-J^{(n)}\right|}{\left(J^{(n+1)}+J^{(n)}\right) / 2}<\xi .
$$

We use a heuristic value of the tolerance parameter, $\xi=0.001$, for both the $l_{1}$-norm and Cauchy norm DRT computation.

The sparse solution for vectors of $W$ at each frequency, i.e., $W\left(s, \omega_{i}\right)$, can thus be obtained by the iterative reweighting scheme. Note that our recent study has confirmed that such a strategy can also be used for the matrix sparsity problem achieving a more accurate dispersion curve extraction. ${ }^{8}$

\section{EXPERIMENTS}

Experiments are achieved using an array transducer consisting of 5 rectangular emitters and 24 rectangular receivers (Vermon, Tours, France) associated with a specific driving electronic device (Althaïs, Tours, France). The pitch of the array transducer is $0.8 \mathrm{~mm}$ and the length and width of each rectangular element are 0.8 and $8 \mathrm{~mm}$, respectively. The central frequency is $1 \mathrm{MHz}$ and the $-6 \mathrm{~dB}$ bandwidth goes from 0.5 to $1.6 \mathrm{MHz}{ }^{15}$

Experiments are carried out on a $1.3 \mathrm{~mm}$ thick bonemimicking plate (Sawbones, Pacific Research Laboratory Inc., Vashon, WA) and an ex vivo human radius whose cortical thickness measured by micro-computed tomography (XtremCT, Scanco Medical, Bruttisellen, Switzerland) was found to be $2.5 \mathrm{~mm}$. Ultrasound gel (Aquasonic, Parker
Labs, Inc., Fairfield, NJ) is used to ensure the coupling between the probe and the sample. Both the bone-mimicking phantom and the human radius are considered to be transversely isotropic elasticity. ${ }^{52}$ The material characteristics of the specimens are listed in Table I. $V_{T}$ is the shear velocity, and $V_{L \|}$ and $V_{L \perp}$ are the pure compression bulk wave velocities in the direction parallel and normal to the direction of the fibers (bone-mimicking material) or to the long axis of the bone (human radius). The density and thickness are denoted by $\rho$ and $h$. Typical values for human radius density, shear, and longitudinal velocity, derived from the literature, are used for computation of the theoretical dispersion curves according to the Rayleigh-Lamb equation. ${ }^{52}$

\section{RESULTS}

The results include synthetic signals of guided waves, multimodal signals measured from the plate model, and the guided signals obtained from ex vivo human radius. The method can be generalized to estimate any parameters related to the dispersion transfer function. As ultrasonic guided waves have been used to characterize the waveguide property, such as the thickness and elasticity, etc., the results are presented for the case of thickness evaluation.

\section{A. Synthetic signals of guided waves}

\section{Results of DRT}

According to Eq. (1), a wideband $\left(0<k \leq 4 \mathrm{rad} \mathrm{mm}^{-1}\right.$, $0<f \leq 2 \mathrm{MHz}$ ) mode $\mathrm{S} 0$ signals on a $1 \mathrm{~mm}$-thick plate phantom were synthesized with the known dispersion curves and excitation [see Fig. 2(a)]. Figure 2(b) depicts the $(\tau, s)$ result [signal-to-noise ratio $(\mathrm{SNR})=\infty$ ] using the fundamental $\tau-s$ transform, i.e., back-projection operator in Eq. (8a), in which the dispersion curve of the S0 mode is used to build the DRT operator $L$. Figure $2(\mathrm{c})$ shows the $(x, t)$ signals reconstructed from the $(\tau, s)$ result in Fig. 2(b) using the forward-projection operator defined in Eq. (8b).

As shown in Fig. 2(d), a Gaussian random noise is then added into the synthetic array signals with a SNR of $0 \mathrm{~dB}$. The SNR is defined as the ratio between the mean powers of the signal and the noise. Since the durations of the dispersive signals vary on propagation distance, all 0-points are removed from the simulated signals for computing the corresponding mean signal power, which ensures both signal and noise can be measured with an equivalent number of points.

The plate thickness value can be estimated from the maximum of the $(\tau, s)$ energy distribution. As noted in the black dotted line in Figs. 2(b) and 2(e), the estimated

TABLE I. Density, thickness, shear, and longitudinal velocities of the specimens.

\begin{tabular}{lcccc}
\hline \hline $\begin{array}{l}\text { Specimens } \\
\text { Bone-mimicking }\end{array}$ & $\rho\left(\mathrm{g} \mathrm{cm}^{-3}\right)$ & $h(\mathrm{~mm})$ & $\mathrm{V}_{\mathrm{T}}\left(\mathrm{mm} \mu \mathrm{s}^{-1}\right)$ & $V_{L}\left(\mathrm{~mm} \mu \mathrm{s}^{-1}\right)$ \\
$\begin{array}{l}\text { plate } \\
\begin{array}{l}\text { Human radius } \\
\text { specimen }\end{array}\end{array}$ & 1.3 & 1.62 & $\begin{array}{c}\left(V_{L \|}, V_{L \perp}\right) \\
=(3.57,2.91)\end{array}$ \\
\hline \hline
\end{tabular}


thickness is $0.97 \mathrm{~mm} \quad(\mathrm{SNR}=\infty)$ and $0.97 \mathrm{~mm} \quad(\mathrm{SNR}$ $=0 \mathrm{~dB}$ ), respectively, and the exact value is $1 \mathrm{~mm}$; the intercepts noted in the red dotted line are both $10 \mu \mathrm{s}$.

The normalized root-mean-square error (NRMSE) is used to measure the difference between the reconstructed signals $g^{\prime}(x, t)$ and the original signal $g(x, t)$. The definition of NRMSE is

$$
\begin{aligned}
& \operatorname{NRMSE}\left(g^{\prime}, g\right) \\
& =\sqrt{\frac{\sum_{x_{i}=1}^{N_{x}} \sum_{t_{j}=1}^{N_{t}}\left|g^{\prime}\left(x_{i}, t_{j}\right)-g\left(x_{i}, t_{j}\right)\right|^{2}}{N_{x} * N_{t}} /\left(g_{\max }-g_{\min }\right),}
\end{aligned}
$$

where $g_{\max }$ and $g_{\min }$ correspond to the maximum and minimum values of $g(x, t)$.

Compared to the original synthetic S0 mode signals [see Fig. 2(a)], the NRMSE of the DRT-based reconstruction is $0.066(\mathrm{SNR}=\infty)$ and $0.092(\mathrm{SNR}=0 \mathrm{~dB})$, respectively. This simple example illustrates that using the fundamental back-projection $(\tau, s)$ operator $L^{H}$, multichannel dispersive and single-mode signals can be mapped in the $(\tau, s)$ field as a localized region with significant energy concentration, and the forward-projection $(\tau, s)$ operator $L$ provides an efficient way for single mode reconstruction in the $(x, t)$ domain. To improve the resolution and achieve a better reconstruction, the performance of least-square $\tau-s$ transform and highresolution $\tau-s$ transform is investigated in Sec. IV A 2.

\section{Results of least-square DRT transform and high-resolution DRT transform}

As shown in Fig. 3, the signals of single mode S0 in Fig. 2 are used to compare the performance of the least- squares DRT and high-resolution DRT. The S0 mode $(\tau, s)$ operator pair is the same as the fundamental DRT method. However, three different penalty strategies are involved to solve the inverse problem in Eq. (9), i.e., $l_{2}$-norm based least-squares DRT method and high-resolution DRT method using $l_{1}$-norm and Cauchy norm. It is illustrated that both the least-square solution and the high resolution DRT method enables to map the multichannel dispersive and single-mode signals in the $(\tau, s)$ field as a localized region with significant energy concentration. Compared with the synthetic S0 mode signals [see Fig. 2(a)], when $\mathrm{SNR}=\infty$, the NRMSEs of the DRT-based reconstruction using the $l_{2}$-norm, $l_{1}$-norm, and Cauchy norm are 0.029 , 0.025 , and 0.009 , respectively; when $\mathrm{SNR}=0 \mathrm{~dB}$, the NRMSEs of the reconstruction using the $l_{2}$-norm, $l_{1}$-norm, and Cauchy norm are $0.059,0.088$, and 0.14 , respectively. When $\mathrm{SNR}=\infty$ and $\mathrm{SNR}=0 \mathrm{~dB}$, the thickness estimates are all $0.98 \mathrm{~mm}$. It illustrates that compared with the fundamental DRT method, the least-square and high-resolution DRT methods enable to filter the noise in the $(\tau, s)$ field with a more accurate reconstruction of the dispersive signals. Furthermore, high-resolution DRT methods enable to achieve a better thickness resolution than the least-squares DRT method. But there is no significant improvement of the thickness estimation in the example.

\section{Finite aperture limitation and DRT resolution}

The resolution is analyzed with varying aperture size and different adjoint inverse strategies. As shown in Fig. 4(a), it can be found that the peaks of energy function obtained at $\tau=10 \mu$ s all locate close to the true thickness value of $1 \mathrm{~mm}$. However the width of the main lobe decreases as the channel number increases with different resolutions in the thickness domain. For example, the $-3 \mathrm{~dB}$ lobe width of the 8 - and 128-channel signals are
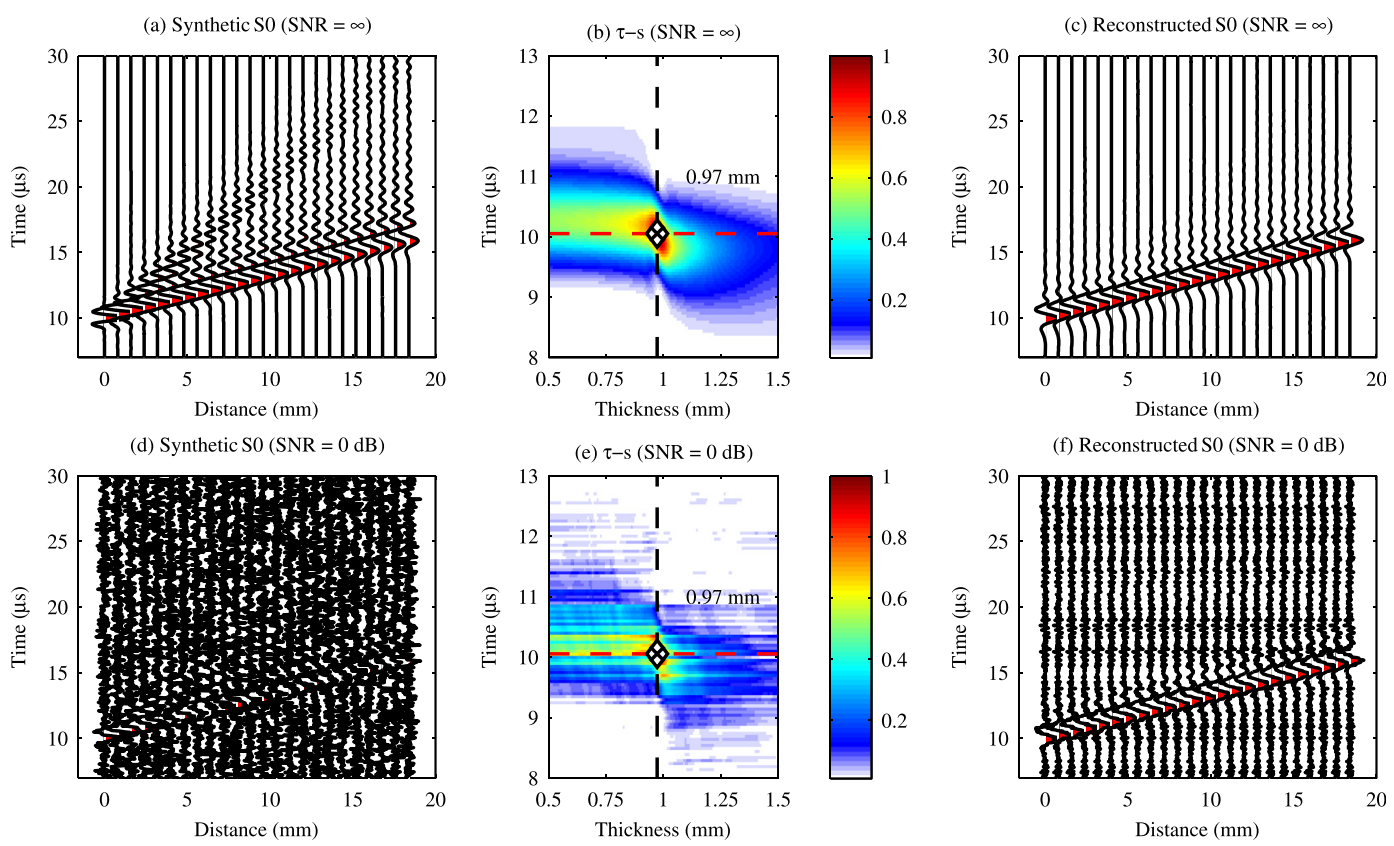

FIG. 2. (Color online) Example of fundamental DRT. Synthetic S0 mode signals $(\mathrm{SNR}=\infty)$, (a) array signals, (b) $(\tau, s)$ distributions, and (c) reconstructed signals. Synthetic S0 mode signals (SNR $=0 \mathrm{~dB}),(\mathrm{d})$ array signals, $(\mathrm{e})(\tau, s)$ distribution, and (f) reconstructed signals. 
(a) $\tau$-s $\left(I_{2}\right)(\mathrm{SNR}=\infty)$

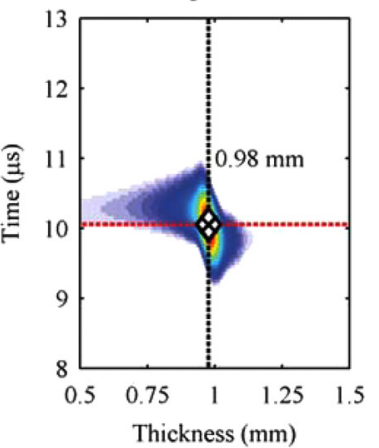

(d) Reconstructed S0, $\tau$-s $(l)(\mathrm{SNR}=\infty)$

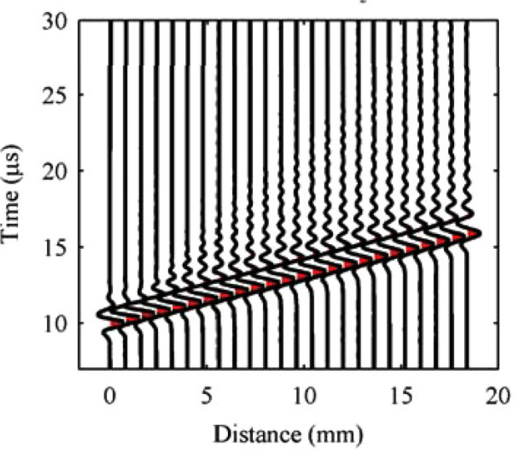

(g) $\tau$-s $\left(I_{2}\right)(\mathrm{SNR}=0 \mathrm{~dB})$

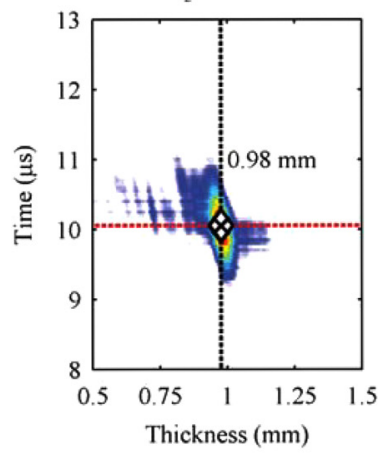

(j) Reconstructed signals $\tau-\mathrm{s}\left(I_{2}\right)(\mathrm{SNR}=0 \mathrm{~dB})$

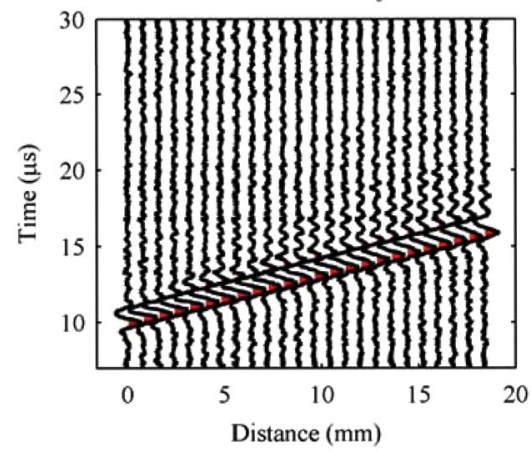

(b) $\tau-\mathrm{s}\left(l_{l}\right)(\mathrm{SNR}=\infty)$

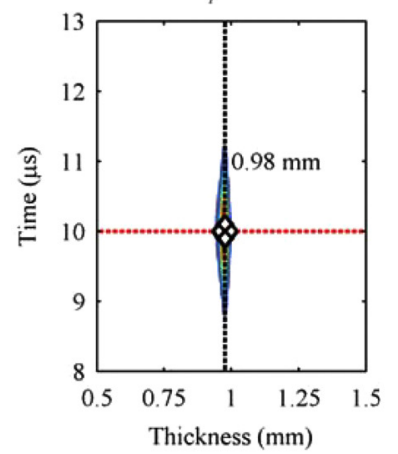

(e) Reconstructed $\mathrm{SO}, \tau$-s $(l)(\mathrm{SNR}=\infty)$

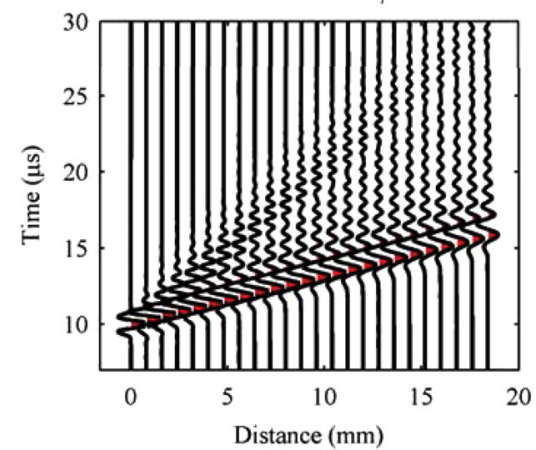

(h) $\tau-\mathrm{s}(l)(\mathrm{SNR}=0 \mathrm{~dB})$
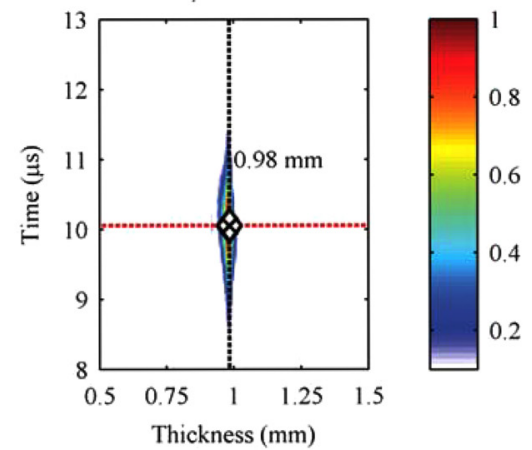

(k) Reconstructed signals $\tau$-s $\left(I_{I}\right)(\mathrm{SNR}=0 \mathrm{~dB})$

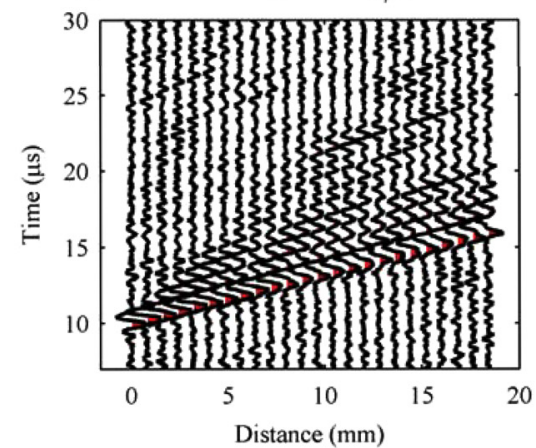

(c) $\tau$-s (Cauchy) $(\mathrm{SNR}=\infty)$

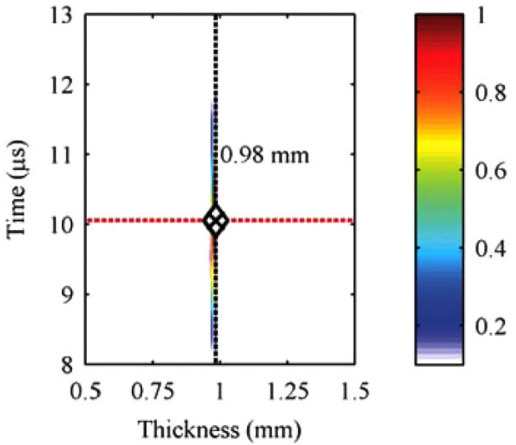

(f) Reconstructed S0, $\tau$-s (Cauchy) $(\mathrm{SNR}=\infty$ )

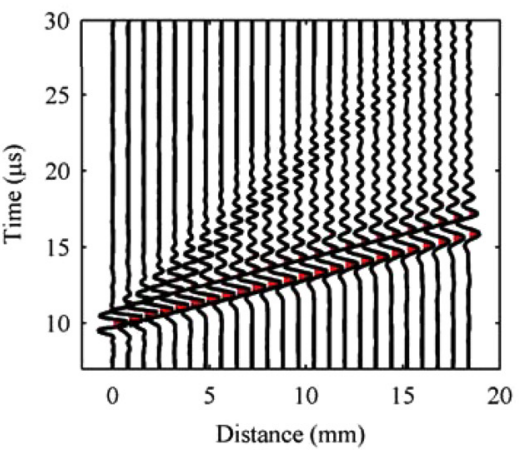

(i) $\tau$-s (Cauchy) $(\mathrm{SNR}=0 \mathrm{~dB})$

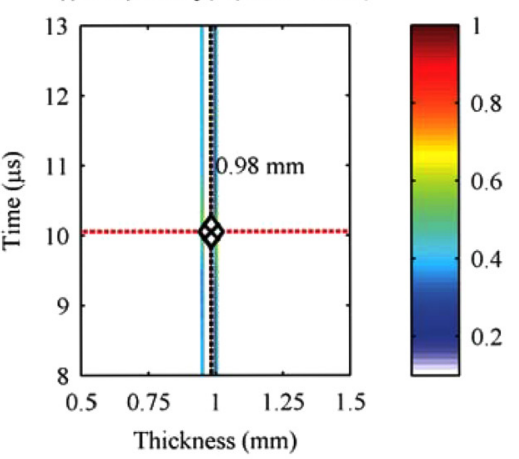

(1) Reconstructed signals $\tau$-s (Cauchy) (SNR $=0 \mathrm{~dB})$

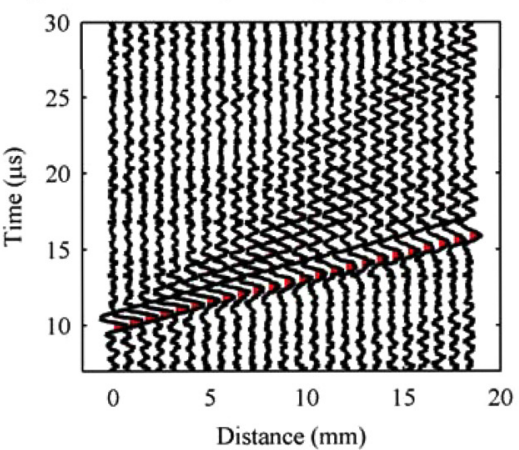

FIG. 3. (Color online) Comparison of different penalty strategies using the synthetic S0 mode signals. (a)-(f) Results obtained when SNR $=\infty$, the $(\tau, s)$ distributions using $l_{2}$-norm, $l_{1}$-norm, and Cauchy norm are presented in (a)-(c), respectively, (f)-(h) are the reconstructed signals from the ( $\left.\tau, s\right)$ distributions of the $l_{2}$-norm, $l_{1}$-norm, and Cauchy norm. (g)-(l) Results obtained when SNR $=0 \mathrm{~dB}$, the $(\tau, s)$ distributions based on $l_{2}$-norm, $l_{1}$-norm, and Cauchy norm are presented in (g)-(i), respectively, and (j)-(l) are the reconstructed signals from the corresponding $(\tau, s)$ distributions.

0.19 and $0.05 \mathrm{~mm}$, respectively. Such a phenomenon, that the parameter estimation resolution is dependent on the aperture size, i.e., probe length, is the so-called finite aperture limitation. As shown in Fig. 4(b), using the fundamental DRT method, the average $-3 \mathrm{~dB}$ lobe-width is $1.11 \mu \mathrm{s}$ and the aperture size has no significant impact on the time resolution. Figures 4(c) and 4(d) compare the energy functions using different back-projection DRT operators. In comparison to the fundamental back-projection $\tau-s$ operator, the penalized operators significantly improve the 
(a)

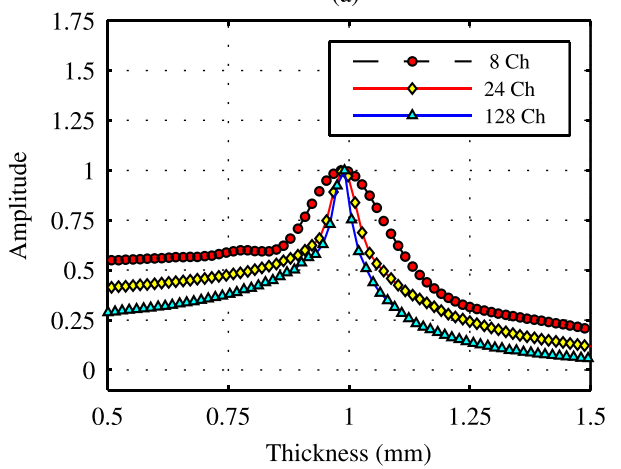

(c)

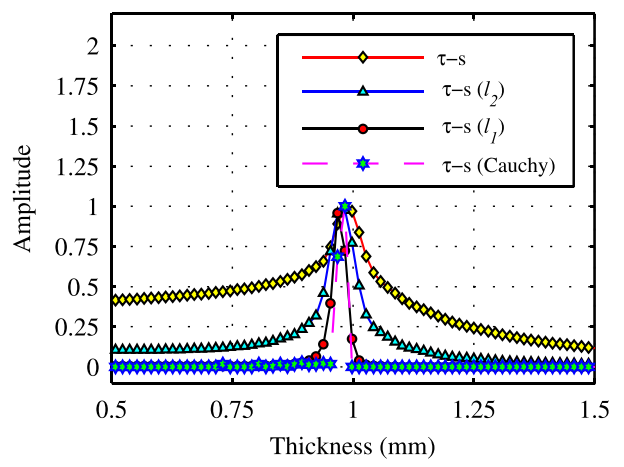

(b)

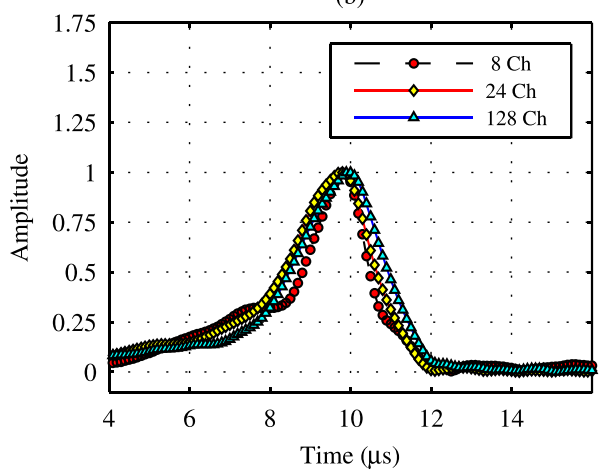

(d)

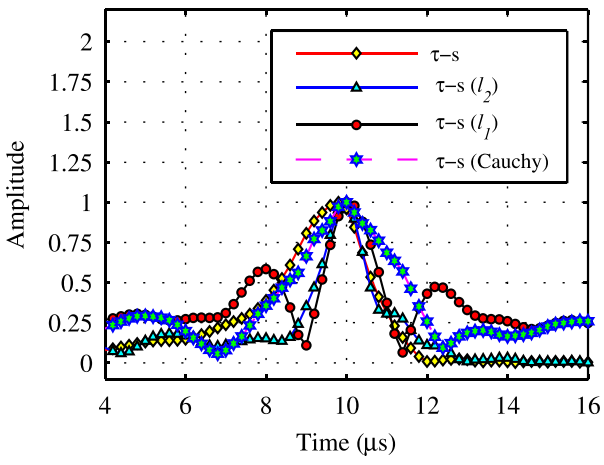

FIG. 4. (Color online) Resolution analysis using the signals of synthetic SO mode $(\mathrm{SNR}=\infty)$, and plate thickness is $1 \mathrm{~mm}$. The fundamental DRT method with varying probe sizes, where the pitch was fixed as $0.8 \mathrm{~mm}$, but with different channel-numbers, i.e., 8, 24, and 128. (a) and (b) show the normalized energy functions obtained at $\tau=10 \mu \mathrm{s}$ and $s=1 \mathrm{~mm}$, respectively. (c) and (d) show the normalized energy functions obtained at $\tau=10 \mu$ s and $s=1 \mathrm{~mm}$, respectively, but with different adjoint inverse strategies ( $l_{2}$-norm, $l_{1}$-norm, and Cauchy norm), where the channel number was fixed as 24 . energy function resolution for thickness estimation. The $-3 \mathrm{~dB}$ main lobe-widths in thickness domain are $0.05 \mathrm{~mm}$ ( $l_{2}$-norm), $0.03 \mathrm{~mm}\left(l_{1}\right.$-norm), and $0.02 \mathrm{~mm}$ (Cauchy norm), and the $-3 \mathrm{~dB}$ main temporal lobe-widths are $0.70 \mu \mathrm{s}$ ( $l_{2}$-norm), $0.80 \mu \mathrm{s}$ ( $l_{1}$-norm), and $1.2 \mu \mathrm{s}$ (Cauchy norm). The time resolutions of $l_{2}$-norm and $l_{1}$-norm based DRT results are better than that of the fundamental $\tau-s$ operator, but the temporal resolution of the Cauchy norm based DRT is worse than the fundamental DRT method. It seems that the sparse penalized operator enhances the side-lobe influence, with a larger ambiguity for arrival time determination. It denotes that there is actually an inherent trade-off between the resolutions of arrival time and model parameter. Akin to the uncertainty principle in quantum mechanics, in the case of the DRT method, the more precise physical model parameter is achieved, less precise its arrival time can be known, and vice versa.

\section{Multimode separation and thickness estimation using the DRT transform}

As shown in Fig. 5, array signals of three wideband modes $\mathrm{A} 0, \mathrm{~S} 0$, and $\mathrm{A} 1$ are synthesized with $0<k \leq 4 \mathrm{rad} \mathrm{mm}^{-1}$, $0<f \leq 2 \mathrm{MHz}$. In this case, the three mode signals are overlapped in both time and frequency domains. A Gaussian random noise is added with a SNR of $5 \mathrm{~dB}$.

Compared to the noise-free synthetic signals of A0, S0, and A1 modes [see Figs. 5(c) and 5(d)], the NRMSEs of the DRT-based reconstruction using the $l_{1}$-norm are 0.033 , 0.061 , and 0.06 , respectively. The estimated values of the thickness are 1.07, 0.97, and $0.97 \mathrm{~mm}$, respectively. This example illustrates that the high-resolution DRT method can be used to filter the noise and to extract the individual mode components from the multimode dispersive signals. The thickness estimation error of the A0 mode is relatively larger than those of modes S0 and A1.

\section{DRT based missing channel recovering}

In practice, some channels of the array-signals can be poorly measured due to improper coupling or different sensitivity of a different receiver. The multimodal signals plotted in Fig. 5(b) are used to testify the DRT method for recovering the missing channels. Each set of array-signals of modes A0, S0, and A1 were reconstructed individually using the corresponding $l_{1}$-norm-based $\tau-s$ operators. Figure 6 presents the reconstructed single-mode signals as well as the multimodal signals which are obtained by summing together the individual modes given in Figs. 6(a)-6(c). Compared to the original noise-free signals in Fig. 5, the NRMSEs of the DRT-based A0, S0, and A1 reconstructions are 0.039, 0.064, and 0.059 , respectively, resulting in a general NRMSE of 0.052 for the multimodal signals.

\section{Model mismatch}

The impact of model mismatch is investigated. As shown in Figs. 7(c) and 7(f), two mismatched dispersion curves of the S0 mode are used to compute the $(\tau, s)$ distributions and to reconstruct the temporal signals. To simplify the physical model, we assumed that the stiffness coefficients can be properly predicted by the cortical porosity ${ }^{54}$ so that the dispersion curves can be obtained. Our original dispersion curves are computed by using a cortical porosity value of $10 \%$, which is used as a normal value of the cortical bone. On the contrary, the mismatched models, i.e., dispersion curves 
(a) Multimode $(\mathrm{SNR}=\infty)$

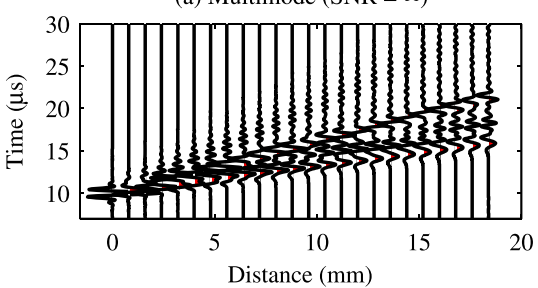

(c) $\mathrm{A} 0(\mathrm{SNR}=\infty)$

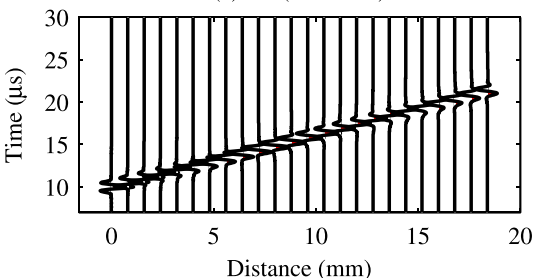

(f) $\tau-\mathrm{s}\left(\mathrm{A} 0, l_{l}\right)(\mathrm{SNR}=5 \mathrm{~dB})$
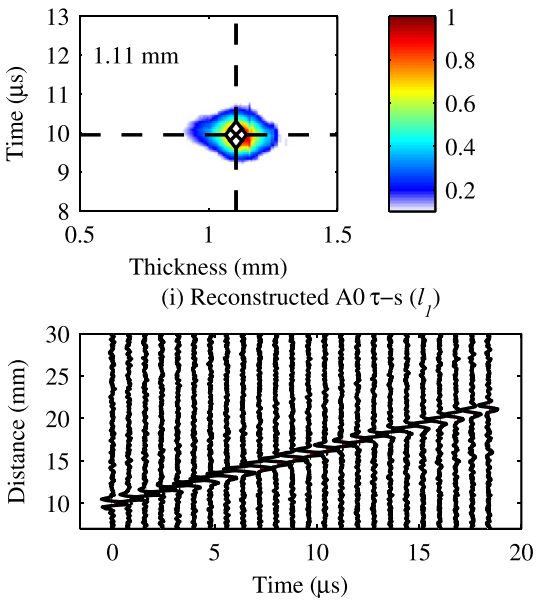

(d) $\mathrm{S} 0(\mathrm{SNR}=\infty)$

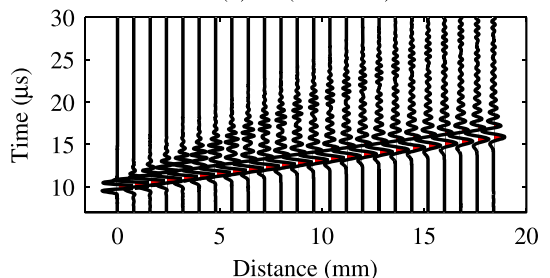

(g) $\tau-\mathrm{s}\left(\mathrm{S} 0, l_{l}\right)(\mathrm{SNR}=5 \mathrm{~dB})$
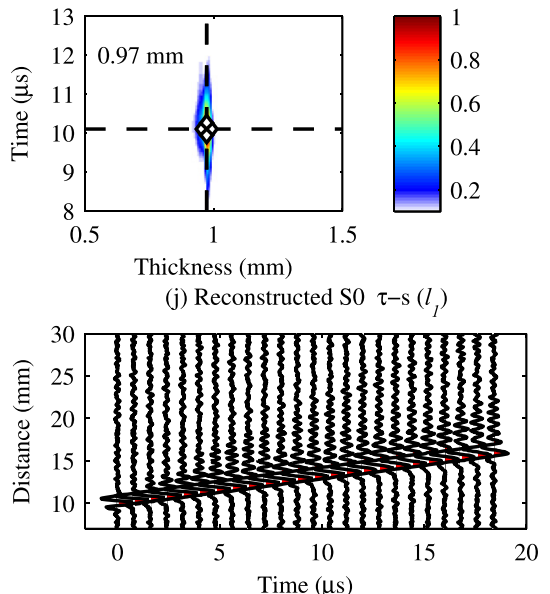

(b) Multimode $(\mathrm{SNR}=5 \mathrm{~dB})$

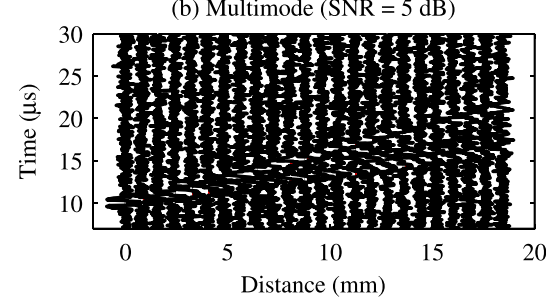

(e) A1 $(\mathrm{SNR}=\infty)$

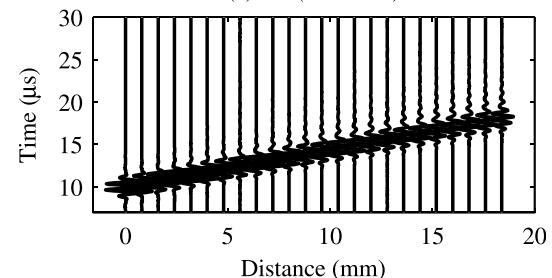

(h) $\tau-\mathrm{s}\left(\mathrm{A} 1, l_{l}\right)(\mathrm{SNR}=5 \mathrm{~dB})$
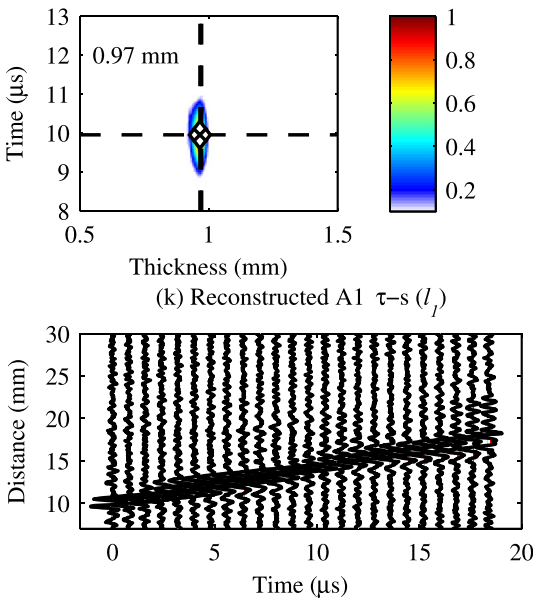

FIG. 5. (Color online) Individual mode extraction using the DRT method. Synthetic signals of A0, S0, and A1 modes (a) original signals, (noise free, $\mathrm{SNR}=\infty)$, (b) with additional Gaussian noise (SNR $=5 \mathrm{~dB})$, (c)-(e) present the noise-free signals of modes A0, S0, and S1, respectively. (f)-(h) display the $(\tau, s)$ results of modes A0, S0, and A1 obtained using the known dispersion curves, where only the $l_{1}$-norm is considered. (i)-(k) display the reconstructed $(x, t)$ signals of each mode after $\tau-s$ forward-projection.

$k_{s 0+}$ and $k_{s 0-}$, were obtained by using the porosity values of $5 \%(\mathrm{~S} 0+)$ and $15 \%(\mathrm{~S} 0-)$. As shown in Figs. 7(a) and 7(d), the thickness estimates obtained by using $k_{s 0+}$ and $k_{s 0-}$ are 1.09 and $1.15 \mathrm{~mm}$, respectively. Compared to the original signals [see Fig. 2(a)], the NRMSEs of the reconstructions obtained by using $k_{s 0+}$ and $k_{s 0-}$ are 0.12 and 0.26 , respectively. Figures 7(c) and 7(f) illustrate that because of the model mismatch, the extracted energy trajectories are distorted and poorly recovered. Similar tests are carried out on the noise-free synthetic signals. Corresponding thickness estimates are $1.09 \mathrm{~mm}\left(k_{s 0+}\right)$ and $1.01 \mathrm{~mm}\left(k_{s 0_{-}}\right)$with reconstructed NRMSEs of $0.07\left(k_{s 0+}\right)$ and $0.01\left(k_{s 0-}\right)$, respectively.

\section{B. Phantom study}

Cortical thickness of long bones can be used as a biomarker of cortical bone strength. ${ }^{15}$ We use the signals measured in a bone-mimicking plate to testify the capability of the proposed DRT method for thickness estimation and individual mode extraction.

Figure 8(a) shows the multichannel signals in the $1.3 \mathrm{~mm}$ thick bone phantom. Figures $8(\mathrm{~b})$ and $8(\mathrm{c})$ depict the $(k, f)$ energy distributions computed using 2DFT and Cauchy-norm based S-SVD. In comparison to the dispersion curves (solid and dashed lines corresponding to antisymmetric and symmetric modes, respectively), three fundamental modes $\mathrm{A} 0, \mathrm{~S} 0$, and A1 can be identified in the $(k, f)$ domain. Figures $8(\mathrm{~d})-8(\mathrm{f})$ depict the $(\tau, s)$ distributions of the $\mathrm{A} 0, \mathrm{~S} 0$, and $\mathrm{A} 1$ modes obtained by using the $l_{1}$-norm based DRT method. The thickness estimates obtained from the maximum of the $(\tau, s)$ energy distributions of the $\mathrm{A} 0, \mathrm{~S} 0$, and $\mathrm{A} 1$ modes are $1.27,1.48$, and $1.42 \mathrm{~mm}$, respectively. The mode overlapping and sidelobe in the $(\tau, s)$ domain could be suppressed with a better model fitting, which is fulfilled by optimizing the sparsity of the high-resolution DRT method. The error of thickness estimation could result from the model mismatch as well as the mode ambiguity and poor SNR.

Figures 9(a)-9(c) show the reconstructed A0, S0, and A1 signals by using the $l_{1}$-norm based DRT method. In Figs. 9(d)-9(f), the results of 2D-FT $(k, f)$ energy distributions illustrate that the individual signals of each mode can be obtained without overlapping. Please note that the S-SVD method normalizes the orthogonal basis in the wavenumber- 
(a) Reconstructed A0 $\tau-\mathrm{s}\left(l_{l}\right)$

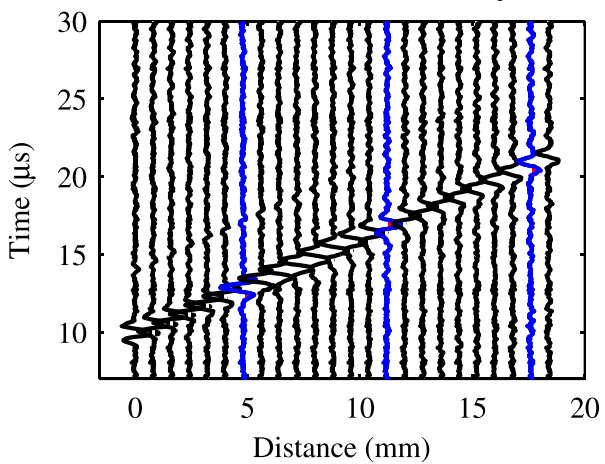

(c) Reconstructed A1 $\tau-\mathrm{s}\left(l_{l}\right)$

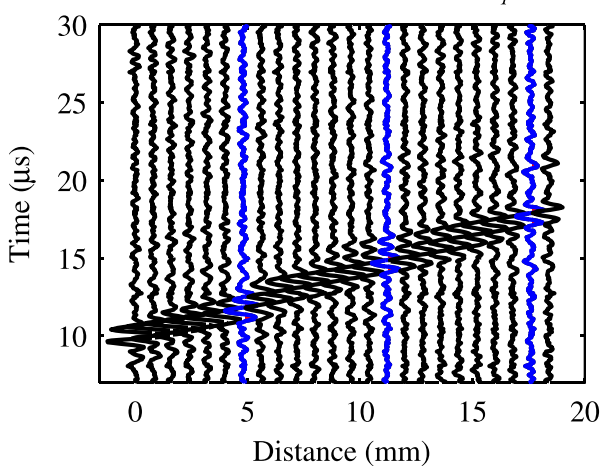

(b) Reconstructed S0 $\tau-\mathrm{s}\left(l_{1}\right)$

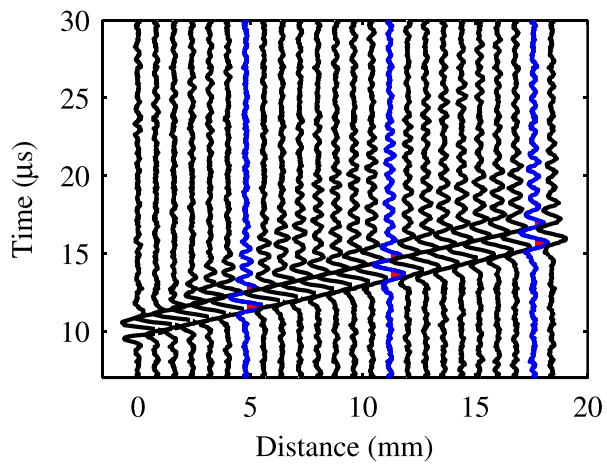

(d) Reconstructed 3 modes $\tau-\mathrm{s}\left(l_{l}\right)$

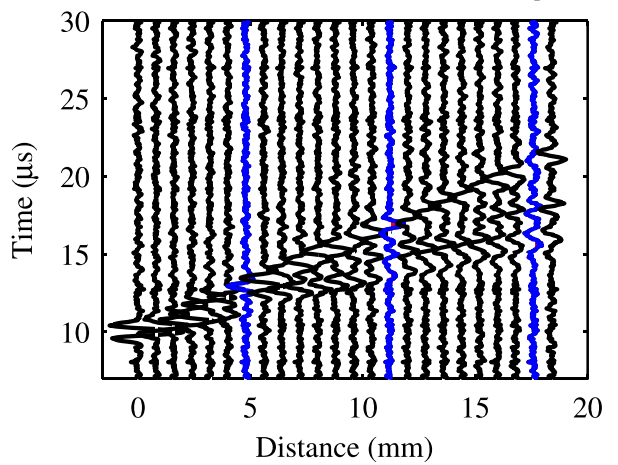

FIG. 6. (Color online) Missing channel reconstruction using the DRT method. The original signals are the same as the signals in Fig. 5(b) (consisting of 3 modes $\mathrm{A} 0$, S0, and $\mathrm{A} 1, \mathrm{SNR}=5 \mathrm{~dB})$, but with three missing channels (channel number $=7,15,23)$. (a) - (c) are the DRT reconstructed signals of A0, S0, and $\mathrm{A} 1$, and (d) is the reconstructed multimode signals. The recovered signals of the three missing channels are highlighted in blue color. frequency domain. Such normalization is performed by using a three-dimension data space (of the multi-emitter, multi-receiver, and time space). However, in this example the DRT method is applied to the data measured using a single emitter and multiple receivers, therefore the SVD procedure cannot be carried out. The extracted $k-f$ energy in Figs. 9(d)-9(f) can be directly compared with the 2 D-FT result in Fig. 8(b).

\section{Analysis of ex vivo signals in a human radius}

The proposed DRT method is applied to measurements on an ex vivo human radius, whose cortical thickness is $2.5 \mathrm{~mm}$.

Figure 10(a) shows the multichannel signals in a $2.5 \mathrm{~mm}$ thick ex vivo human radius. As shown in Figs. 10(b) and 10(c), four fundamental modes A0, S0, A1, and S1 can be observed in the $(k, f)$ domain. At high frequency-thickness
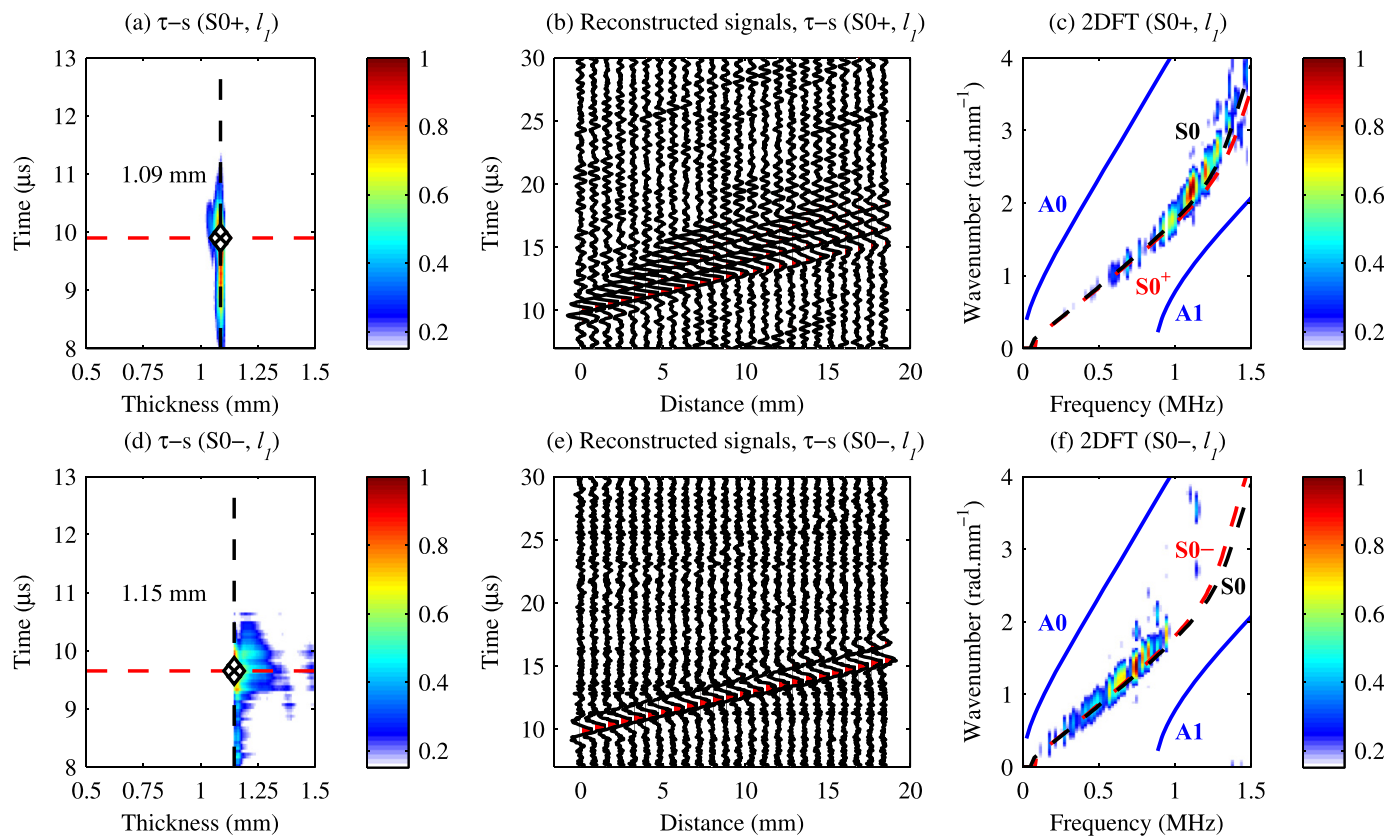

FIG. 7. (Color online) Illustration of the model-mismatch impact using the S0 signals in Fig. 2(d) (SNR $=0 \mathrm{~dB})$. (a)-(c) Results obtained by using the S0+ dispersion curve [red line in (c)], (a) $(\tau, s)$ distribution, (b) reconstructed signals, (c) $(k, f)$ energy distribution. (d)-(f) Results obtained by using the S0-dispersion curve [red line in (f)], (d) $(\tau, s)$ distribution, (e) reconstructed signals, and (f) $(k, f)$ energy distribution. Only the $l_{1}$-norm-based $\tau-s$ operators are considered. The $(k, f)$ energy distributions are computed using 2D-FT. 

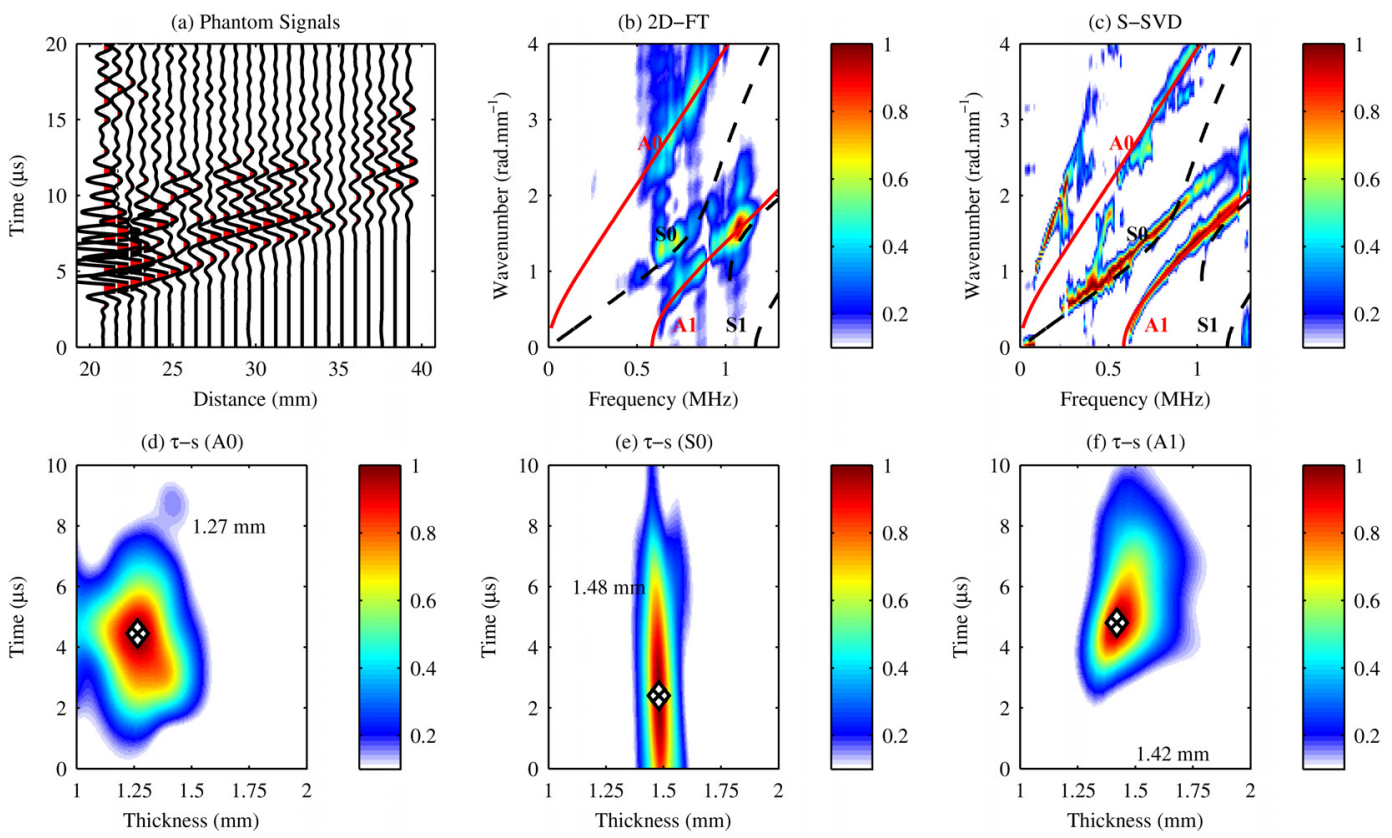

FIG. 8. (Color online) Thickness estimation of a $1.3 \mathrm{~mm}$ thick phantom plate. (a) Multichannel signals, (b) and (c) $(k, f)$ energy distributions computed using 2D-FT and Cauchy-norm based S-SVD method. (d)-(f) $(\tau, s)$ distributions of A0, S0, and A1 modes by using the $l_{1}$-norm based $\tau-s$ transform.

range, the $\mathrm{A} 0$ mode propagates at a constant phase velocity without significant dispersion and is not very sensitive to the cortical bone thickness. Consequently, fundamental modes S0, A1, and S2 are used for thickness estimation. Figures $10(\mathrm{~d})-10(\mathrm{f})$ depict the $(\tau, s)$ distributions of the S0, A1, and S2 modes obtained using the $l_{1}$-norm based DRT method. The thickness estimates obtained from the maximum of the $(\tau, s)$ energy distributions of the $\mathrm{S} 0, \mathrm{~A} 1$, and $\mathrm{S} 2$ modes are $2.19,2.31$, and $2.11 \mathrm{~mm}$, respectively.

Figures 11(a)-11(c) show the reconstructed S0, A1, and S2 signals. In Figs. 11(d)-11(f), good agreements are observed between the $(k, f)$ energy distributions and the corresponding dispersion curves of the reconstructed S0, A1, and S2 modes.

\section{DISCUSSION}

Motivated by many merits of the classical RT method, e.g., reversibility, denoising capability, missing-data reconstruction, and weak data detection, etc., and particularly its facility of space projection, in this study the adjoint pair of DRT are proposed, which is established by the integration operators between the dispersive Radon field and the $(x, t)$ domain. The proposed DRT method allows to selectively
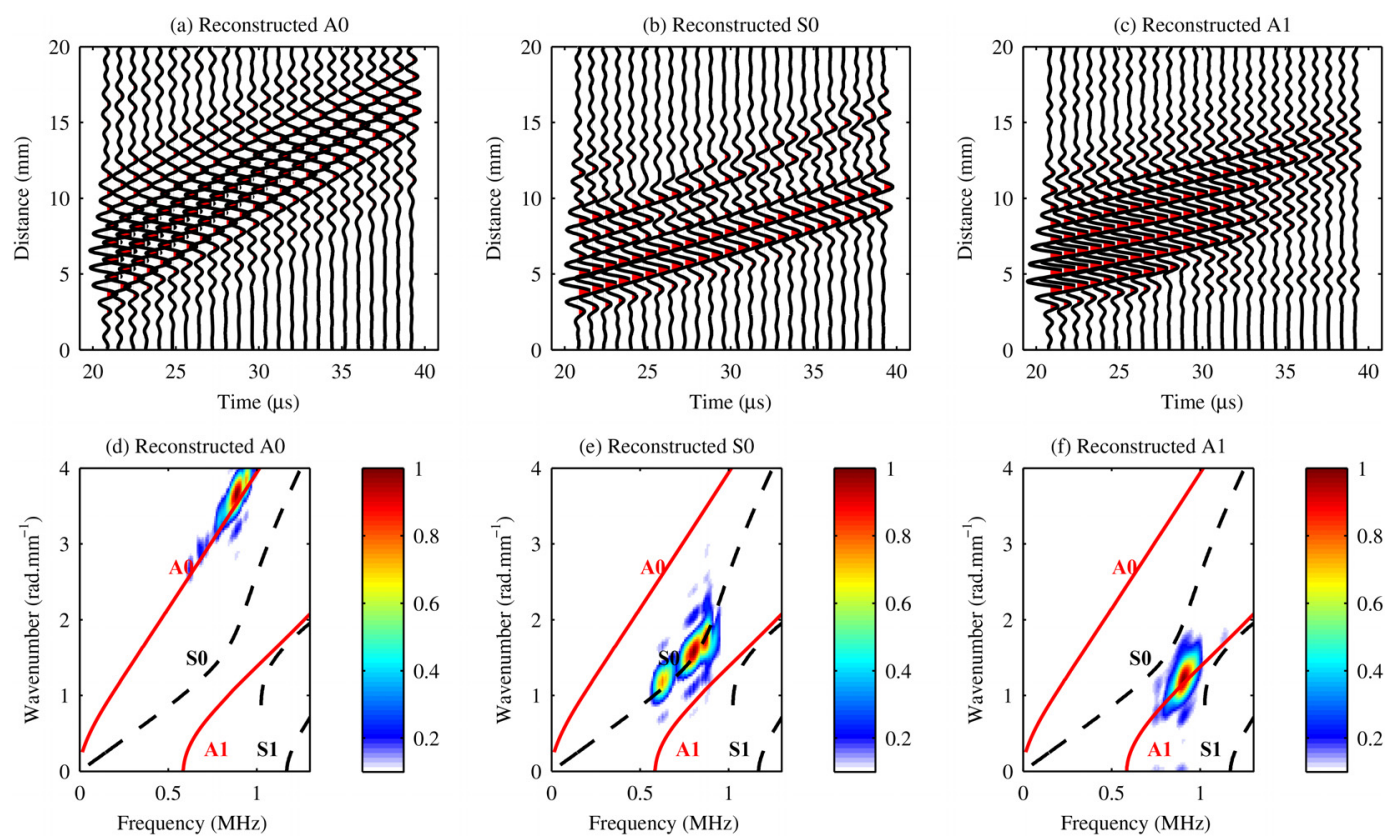

FIG. 9. (Color online) Individual mode reconstruction for a $1.3 \mathrm{~mm}$ thick phantom. (a)-(c) depict the reconstructed A0, S0, and A1 signals from the ( $\tau, s)$ distributions by using the $l_{1}$-norm based DRT. (d)-(f) display the 2D-FT $(k, f)$ energy distribution of the reconstructed A0, S0, and A1 modes, respectively. 

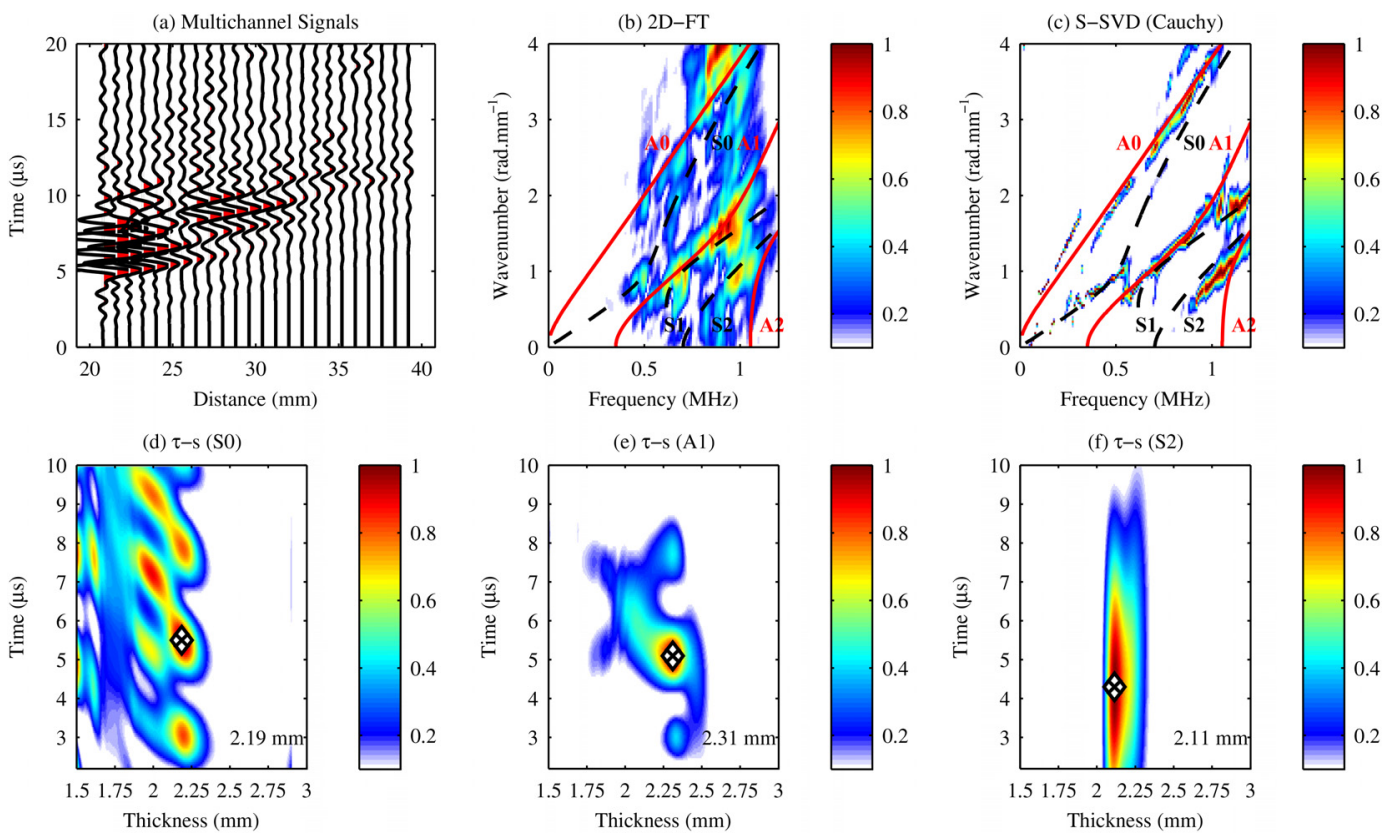

FIG. 10. (Color online) Thickness assessment of a $2.5 \mathrm{~mm}$ thick ex vivo human radius. (a) Multichannel signals, (b) and (c) display the ( $k, f)$ energy distributions computed using 2D-FT and Cauchy-norm based S-SVD method. (d)-(f) are the $(\tau, s)$ distributions of the S0, A1, and S2 modes by using the $l_{1}$-norm based $\tau-s$ transform. Solid and dashed lines corresponding to antisymmetric and symmetric modes, respectively.

map the $(x, t)$ signal of a single mode to the DRT domain as a localized region with energy focusing, which provides an efficient way for mode identification, mode separation, noise filtering, and missing data reconstruction. In addition, such an operator pair straightforwardly integrates the dispersive signal processing and waveguide property estimation. The sparse inversion technique is introduced to filter the noise and suppress the influence of undesired modes, leading to the high-resolution DRT solution. The potential of the proposed method is illustrated using synthetic signals and experimental data.
In classical RT, two parameters, i.e., the intercept $\tau$ and the slope or slowness $p$, are used to describe the line in the $(x, t)$ domain. For highly-dispersive modes, which are usually observed without clear ray-shape wave-packets in the $(x, t)$ domain, they cannot be concentrated in the $(\tau, p)$ field. On the contrary, in the proposed DRT method, wavenumber dispersion transfer function is directly implemented inside the operator. The dispersive signals can thus be mapped into the $(\tau, s)$ field with showing the energy distribution for the parameter of interest, whose maximal can be directly used to estimate the waveguide property. Furthermore, taking
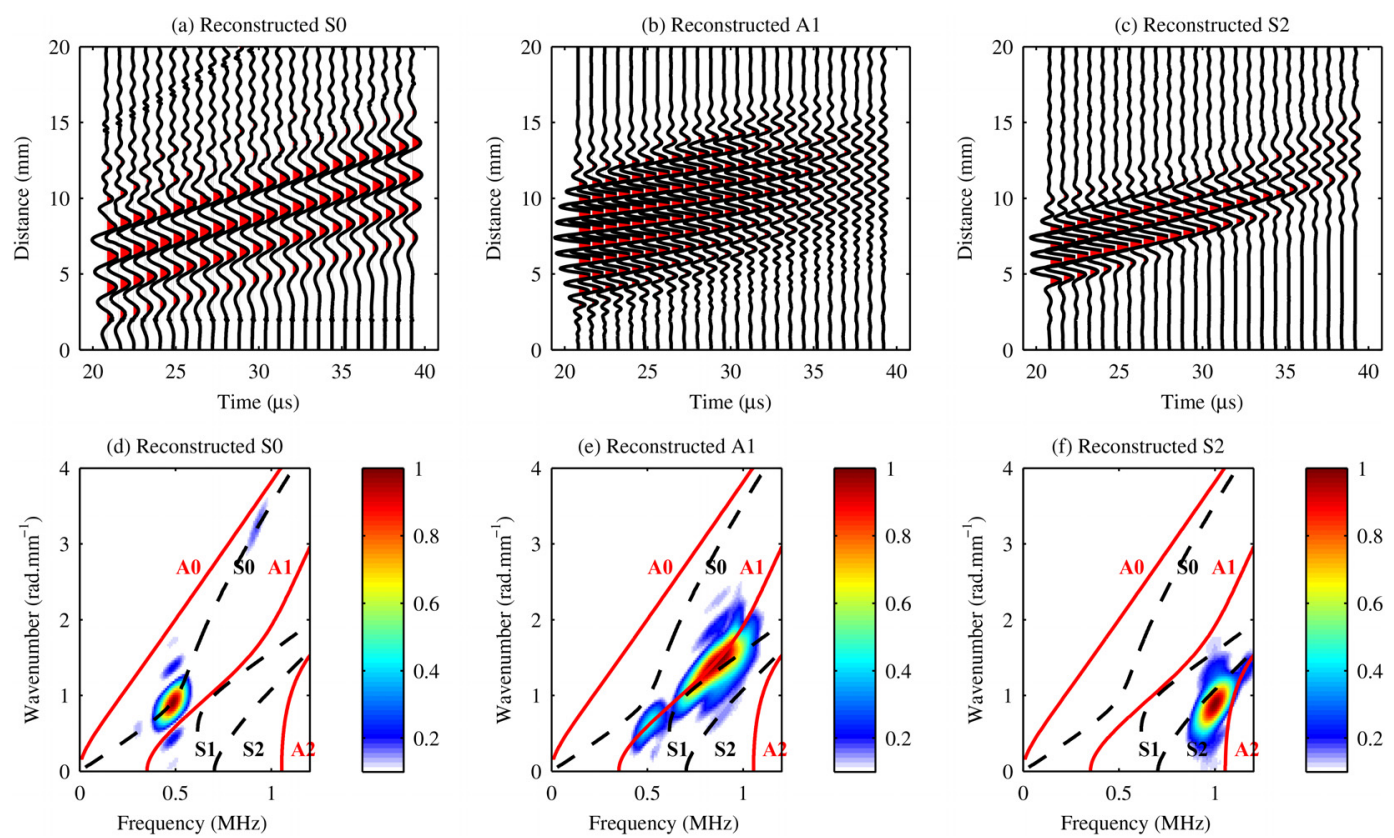

FIG. 11. (Color online) Individual mode reconstruction of a $2.5 \mathrm{~mm}$ thick ex vivo human radius. (a)-(c) are the reconstructed S0, A1, and S2 signals from the $(\tau, s)$ distributions by using the $l_{1}$-norm based $\tau-s$ transform. (d)-(f) display the $(k, f)$ energy distributions of the reconstructed S0, A1, and S2 modes. 
advantage of the sparse penalty, the high-resolution DRT can enforce the sparsity of wideband dispersive modes in the $(\tau, s)$ domain with a better resolution for the parameter estimation.

Tran et al ${ }^{37,53}$ have introduced the classical and highresolution $\mathrm{RT}$ method to analyze ultrasonic guided signals in long bone. They showed that the linear RT method enables enhancing the resolution of the extracted dispersion curves in comparison to the 2D-FT method. However, our group reported that extracting the wideband dispersion curves using the linear RT method remains challenging, ${ }^{38}$ in particular, for low-amplitude multimode signals and for poor SNR, corresponding to ultrasonic guided waves measured in human long cortical bone. Our recently proposed S-SVD method $^{8}$ can significantly enhance the weak modes extraction. However, it is still based on the classical FT pairs, i.e., distance to wavenumber and time to frequency, so that besides the dispersion curves extraction, it cannot directly link the signal space to other parameter space for waveguide characterization. Furthermore, the non-dispersive Radon dictionary $^{37,53}$ is based on the assumption of low-dispersive signal with small velocity variation, which limits its application in the wideband dispersive signal processing. The proposed DRT method seeks another sparse projection on the series of dispersive basis of each individual guided mode, whose propagation field is directly parameterized by the physical property of the waveguide in the DRT domain. Such a property straightforwardly integrates the signal processing to the inverse problem, which can thus be used to solve the challenge for dispersion-based material and structure characterization.

A limitation of the performed study is that we only consider a two dimension DRT space, i.e., intercept and thickness domain. However, depending on different applications, it can be valuable to introduce other parameters into the DRT, which can open the potentials for multi-parameter evaluation, e.g., elasticity, mass density, or longitudinal and shear velocities. ${ }^{54}$ With a full-set of multi-parameter dispersion curves, a high-dimension modal basis with several model parameters can be readily built. Since the proposed DRT method only consists of forward and backward projection pair, an efficient computation for high dimension DRT can be expected. Multimodal guided wave signals in the plate-like structure are presented as examples to demonstrate the feasibility of the proposed DRT method. With the known dispersion curves, the proposed DRT method can be used to estimate the cortical thickness. The results of the simulations and experiments are summarized in Table II.

For different cases, such as the dispersive guided waves in tubular structures or Rayleigh waves, the principles of DRT can be readily extended with different dispersion functions. In the presence of absorption, attenuation is another important issue along with the dispersion, which weakens amplitude and impairs SNR. The attenuated guided waves with complex wavenumber ${ }^{55}$ can be considered in the DRT field as it has been considered in the 2D-FT domain. It has been illustrated that the mode extraction can enhance the feature extraction, which increases the robustness and reliability for damage identification ${ }^{56}$ and tomography. ${ }^{57,58}$ Therefore, many signal processing methods have been proposed to extract the temporal signals for each individual mode in the non-destructive field, for instance 2D filtering in wavenumber-frequency domain, ${ }^{59}$ TFR ridge extraction and reconstruction, ${ }^{60}$ dispersion compensation based mode separation, ${ }^{17,61}$ Chirplet transform, ${ }^{23}$ orthogonality relationbased method, ${ }^{62}$ and joint approximate diagonalization of eigen-matrices algorithm, ${ }^{63}$ etc. With the known dispersion curves, the DRT method provides an efficient way for mode identification and separation, so that it may potentially be helpful for guided waves tomography.

The DRT method involves matrix inversion in the iteration step. On average, 20 times iterations are sufficient to converge to the sparse solution, which suggests that it is capable for the real-time processing.

The DRT method is still dependent on the preknowledge of the dispersion curves. Especially when dispersion curves of some modes are too close to be readily distinguished, the DRT method may not be able to extract the pure modes. As the number and the orders of recorded modes vary in different frequency-thickness ranges, instead of a single mode strategy, multimodal analysis should be considered toward the application of dispersion curve based waveguide evaluation. In addition, the mode sensitivity has impacts on the parameter estimation, which has not been thoroughly discussed in this study.

\section{CONCLUSION}

An original DRT is proposed in this study. The analysis of synthetic signals and experimental data together suggests many merits of the DRT method for processing multichannel dispersive signals. The back-projection DRT operator can map the $(x, t)$ signal of a single mode to the DRT domain as a well localized region, which provides an efficient way for mode identification and mode separation. The forwardprojection DRT operator can map the signals into the space and time $(x, t)$ domain, which allows us to reconstruct the signals, filter the noise, and recover the missing data. The DRT operators directly link the spatial space to the parameter space. This property straightforwardly integrates the signal processing to the inverse problem, which can be used to solve the challenge for dispersion-based material and

TABLE II. Thickness estimation results.

\begin{tabular}{|c|c|c|c|c|c|c|c|}
\hline & & AO & So & $A 1$ & $S 2$ & Mean + Standard Deviation $(\mathrm{mm})$ & True thickness ( $\mathrm{mm}$ ) \\
\hline Simulation & & 1.11 & 0.97 & 0.97 & N/A & $1.01 \pm 0.07$ & 1 \\
\hline \multirow[t]{2}{*}{ Experiments } & Bone-mimicking plate & 1.27 & 1.48 & 1.42 & N/A & $1.39 \pm 0.11$ & 1.3 \\
\hline & Human radius specimen & N/A & 2.19 & 2.31 & 2.11 & $2.20 \pm 0.30$ & 2.5 \\
\hline
\end{tabular}


structure characterization. As an example, in this paper the waveguide thickness has been estimated from the mode energy in the DRT domain. Based on the sparse penalization strategy, a high resolution DRT method is proposed to overcome the finite-aperture problem. An infinite-aperture dispersive velocity gather can thus be achieved. A model mismatch study shows that the DRT method is robust for mode separation and parameter estimation with inaccurate dispersion curves. The invertibility of the proposed DRT and an efficient discrete algorithm implemented in the Fourier domain suggests that the DRT operators can be a potentially generalized solution for dispersive signal processing. To overcome the limitation of single parameter inversion, future work can focus on developing the DRT based inversion of multiple parameters.

\section{ACKNOWLEDGMENT}

The authors benefited from discussions with Dr. Maryline Talmant.

${ }^{1}$ V. C. Chen and H. Ling, "Joint time-frequency analysis for radar signal and image processing," IEEE Signal Proc. Mag. 16, 81-93 (1999).

${ }^{2}$ J. D. Irving and R. J. Knight, "Removal of wavelet dispersion from ground-penetrating radar data," Geophys. 68, 960-970 (2003).

${ }^{3}$ C. P. Oden, M. H. Powers, D. L. Wright, and G. R. Olhoeft, "Improving GPR image resolution in lossy ground using dispersive migration," IEEE Trans. Geosci. Remote 45, 2492-2500 (2007).

${ }^{4} \mathrm{G}$. W. Wornell, "Emerging applications of multirate signal processing and wavelets in digital communications," Proc. IEEE 84, 586-603 (1996).

${ }^{5} \mathrm{~K}$. Popat and K. Zeger, "Robust quantization of memoryless sources using dispersive FIR filters," IEEE Trans. Commun. 40, 1670-1674 (1992).

${ }^{6} \mathrm{~W}$. Sachse and Y. Pao, "On the determination of phase and group velocities of dispersive waves in solids," J. Appl. Phys. 49, 4320-4327 (1978).

${ }^{7}$ W. H. Prosser, M. D. Seale, and B. T. Smith, "Time-frequency analysis of the dispersion of Lamb modes," J. Acoust. Soc. Am. 105, 2669-2676 (1999).

${ }^{8}$ K. Xu, J. Minonzio, D. Ta, B. Hu, W. Wang, and P. Laugier, "Sparse SVD method for high resolution extraction of the dispersion curves of ultrasonic guided waves," IEEE Trans. Ultrason. Ferroelectr. Freq. Control 63, 1514-1524 (2016).

${ }^{9}$ B. Boashash and H. J. Whitehouse, "Seismic applications of the WignerVille distribution," in Proceedings of the International Conference in Systems and Circuits (1986), pp. 34-37.

${ }^{10} \mathrm{G}$. A. McMechan and M. J. Yedlin, "Analysis of dispersive waves by wave field transformation," Geophys. 46, 869-874 (1981).

${ }^{11}$ C. B. Park, "Imaging dispersion curves of surface waves on multi-channel record," SEG Tech. Program Expanded Abstracts 17, 1377-1380 (1998).

${ }^{12}$ L. Wang, Y. Xu, J. Xia, and Y. Luo, "Effect of near-surface topography on high-frequency Rayleigh-wave propagation," J. Appl. Geophys. 116, 93-103 (2015).

${ }^{13}$ C. Chen, J. H. Miller, G. F. Bourdreaux-Bartels, G. R. Potty, and C. J. Lazauski, "Time-frequency representations for wideband acoustic signals in shallow water," in Proceedings of OCEANS 2003 (2003), Vol. 5, pp. SP2903-SP2907.

${ }^{14}$ C. Ioana, A. Jarrot, C. Gervaise, Y. Stephan, and A. Quinquis, "Localization in underwater dispersive channels using the time-frequencyphase continuity of signals," IEEE Trans. Signal Process. 58, 4093-4107 (2010).

${ }^{15}$ Q. Vallet, N. Bochud, C. Chappard, P. Laugier, and J. Minonzio, "In vivo characterization of cortical bone using guided waves measured by axial transmission," IEEE Trans. Ultrason. Ferroelectr. Freq. Control 63, 1361-1371 (2016).

${ }^{16}$ P. Dutta, M. W. Urban, O. P. Le Maitre, J. F. Greenleaf, and W. Aquino, "Simultaneous identification of elastic properties, thickness, and diameter of arteries excited with ultrasound radiation force," Phys. Med. Biol. 60, 5279-5296 (2015).
${ }^{17}$ K. Xu, D. Ta, P. Moilanen, and W. Wang, "Mode separation of Lamb waves based on dispersion compensation method," J. Acoust. Soc. Am. 131, 2714-2722 (2012).

${ }^{18}$ L. Cohen, "Time-frequency distributions-a review," Proc. IEEE 77, 941-981 (1989).

${ }^{19}$ A. Moghaddar and E. K. Walton, "Time-frequency distribution analysis of scattering from waveguide cavities," IEEE Trans. Antennas Propag. 41, 677-680 (1993).

${ }^{20}$ A. Papandreou-Suppappola, R. L. Murray, B. G. Iem, and G. F. Boudreaux-Bartels, "Group delay shift covariant quadratic time-frequency representations," IEEE Trans. Signal Process. 49, 2549-2564 (2001).

${ }^{21}$ J. Bonnel, G. E. G. Le Touz, E. B. Nicolas, and J. Mars, "Power class utilization with waveguide-invariant approximation," IEEE Signal Proc. Mag. 30, 120-129 (2013).

${ }^{22}$ J. Hong, K. H. Sun, and Y. Y. Kim, "Dispersion-based short-time Fourier transform applied to dispersive wave analysis," J. Acoust. Soc. Am. 117, 2949-2960 (2005).

${ }^{23}$ H. Kuttig, M. Niethammer, S. Hurlebaus, and L. J. Jacobs, "Model-based analysis of dispersion curves using chirplets," J. Acoust. Soc. Am. 119, 2122-2130 (2006).

${ }^{24}$ G. L. Touze, B. Nicolas, J. I. Mars, and J. L. Lacoume, "Matched representations and filters for guided waves," IEEE Trans. Signal Process. 57, 1783-1795 (2009).

${ }^{25}$ C. B. Park, R. D. Miller, and J. Xia, "Multichannel analysis of surface waves," Geophys. 64, 800-808 (1999).

${ }^{26}$ J. G. Minonzio, M. Talmant, and P. Laugier, "Guided wave phase velocity measurement using multi-emitter and multi-receiver arrays in the axial transmission configuration," J. Acoust. Soc. Am. 127, 2913-2919 (2010).

${ }^{27}$ C. H. Chapman, "A new method for computing synthetic seismograms," Geophys. J. Int. 54, 481-518 (1978).

${ }^{28}$ J. B. Harley and J. M. F. Moura, "Sparse recovery of the multimodal and dispersive characteristics of Lamb waves," J. Acoust. Soc. Am. 133, 2732-2745 (2013).

${ }^{29}$ W. Zhao, M. Li, J. B. Harley, Y. Jin, J. M. Moura, and J. Zhu, "Reconstruction of lamb wave dispersion curves by sparse representation with continuity constraints," J. Acoust. Soc. Am. 141, 749-763 (2017).

${ }^{30}$ F. L. Courtois and J. Bonnel, "Compressed sensing for wideband wavenumber tracking in dispersive shallow water," J. Acoust. Soc. Am. 138, 575-583 (2015).

${ }^{31}$ A. Dremeau, F. L. Courtois, and J. Bonnel, "Reconstruction of dispersion curves in the frequency-wavenumber domain using compressed sensing on a random array," IEEE J. Ocean. Eng. 42(4), 914-922 (2017).

${ }^{32}$ C. H. Chapman, "Generalized Radon transforms and slant stacks," Geophys. J. Int. 66, 445-453 (1981).

${ }^{33}$ G. Beylkin, "Discrete radon transform," IEEE Trans. Acoust., Speech, Signal Process. 35, 162-172 (1987).

${ }^{34}$ L. Ambrozinski, B. Piwakowski, T. Stepinski, and T. Uhl, "Evaluation of dispersion characteristics of multimodal guided waves using slant stack transform," NDT\&E Int. 68, 88-97 (2014).

${ }^{35}$ J. R. Thorson and J. F. Claerbout, "Velocity-stack and slant-stack stochastic inversion," Geophys. 50, 2727-2741 (1985).

${ }^{36}$ Y. Luo, J. Xia, R. D. Miller, Y. Xu, J. Liu, and Q. Liu, "Rayleigh-wave dispersive energy imaging using a high-resolution linear Radon transform," Pure Appl. Geophys. 165, 903-922 (2008).

${ }^{37}$ T. N. Tran, L. H. Le, M. D. Sacchi, V. Nguyen, and E. H. Lou, "Multichannel filtering and reconstruction of ultrasonic guided wave fields using time intercept-slowness transform," J. Acoust. Soc. Am. 136, 248-259 (2014).

${ }^{38}$ K. Xu, D. Ta, D. Cassereau, B. Hu, W. Wang, P. Laugier, and J. Minonzio, "Multichannel processing for dispersion curves extraction of ultrasonic axial-transmission signals: Comparisons and case studies," J. Acoust. Soc. Am. 140, 1758-1770 (2016).

${ }^{39}$ A. Baggeroer, W. Kuperman, and P. Mikhalevsky, "An overview of matched field methods in ocean acoustics," IEEE J. Ocean. Eng. 18, 401-424 (1993).

${ }^{40} \mathrm{~T}$. Yang, "Effectiveness of mode filtering: A comparison of matched-field and matched-mode processing," J. Acoust. Soc. Am. 87, 2072-2084 (1990).

${ }^{41}$ A. Tolstoy, "Applications of matched-field processing to inverse problems in underwater acoustics," Inverse Probl. 16, 1655-1666 (2000).

${ }^{42}$ J. B. Harley and J. M. Moura, "Data-driven matched field processing for lamb wave structural health monitoring," J. Acoust. Soc. Am. 135, 1231-1244 (2014). 
${ }^{43}$ J. Bonnel, B. Nicolas, J. I. Mars, and S. C. Walker, "Estimation of modal group velocities with a single receiver for geoacoustic inversion in shallow water," J. Acoust. Soc. Am. 128, 719-727 (2010).

${ }^{44}$ J. E. Hipp, "Soil electromagnetic parameters as functions of frequency, soil density, and soil moisture," Proc. IEEE 62, 98-103 (1974).

${ }^{45}$ I. A. Viktorov, Rayleigh and Lamb Waves: Physical Theory and Applications (Plenum Press, New York, 1967).

${ }^{46}$ M. D. Sacchi, "Statistical and transform methods in geophysical signal processing," (2002), pp. 209-253, available at http://carina.fcaglp.unlp. edu.ar/senales/libros/text_book_Sacchi.pdf (Last viewed April 29, 2018).

${ }^{47}$ J. P. Burg, "Maximum entropy spectral analysis," Ph.D. thesis, Stanford University, 1975.

${ }^{48}$ D. Trad, M. D. Sacchi, and T. J. Ulrych, "Latest views of the sparse Radon transform," Geophys. 68, 386-399 (2003).

${ }^{49}$ M. D. Sacchi and T. J. Ulrych, "High-resolution velocity gathers and offset space reconstruction," Geophys. 60, 1169-1177 (1995).

${ }^{50}$ M. D. Sacchi, "Reweighting strategies in seismic deconvolution," Geophys. J. Int. 129, 651-656 (1997).

${ }^{51}$ R. Schultz and Y. J. Gu, "Flexible, inversion-based Matlab implementation of the Radon transform," Comput. Geosci. 52, 437-442 (2013).

${ }^{52}$ J. Foiret, J. G. Minonzio, C. Chappard, M. Talmant, and P. Laugier, "Combined estimation of thickness and velocities using ultrasound guided waves: A pioneering study on in vitro cortical bone samples," IEEE Trans. Ultrason. Ferroelectr. Freq. Control 61, 1478-1488 (2014).

${ }^{53}$ T. N. Tran, K. T. Nguyen, M. D. Sacchi, and L. H. Le, "Imaging ultrasonic dispersive guided wave energy in long bones using linear Radon transform," Ultrasound Med. Biol. 40, 2715-2727 (2014).

${ }^{54}$ N. Bochud, Q. Vallet, T. Bala, H. Follet, J.-G. Minonzio, and P. Laugier, "Genetic algorithms-based inversion of multimode guided waves for cortical bone characterization,” Phys. Med. Biol. 61, 6953-6974 (2016).
${ }^{55}$ J. G. Minonzio, J. Foiret, M. Talmant, and P. Laugier, "Impact of attenuation on guided mode wavenumber measurement in axial transmission on bone mimicking plates," J. Acoust. Soc. Am. 130, 3574-3582 (2011).

${ }^{56} \mathrm{~K}$. Xu, D. Ta, Z. Su, and Z. W. Wang, "Transmission analysis of ultrasonic Lamb mode conversion in a plate with partial-thickness notch," Ultrasonics 54, 395-401 (2014).

${ }^{57}$ K. R. Leonard and M. K. Hinders, "Multi-mode Lamb wave tomography with arrival time sorting," J. Acoust. Soc. Am. 117, 2028-2038 (2005).

${ }^{58}$ J. Rao, M. Ratassepp, and Z. Fan, "Guided wave tomography based on full waveform inversion," IEEE Trans. Ultrason. Ferroelectr. Freq. Control 63, 737-745 (2016).

${ }^{59}$ T. Hayashi and K. Kawashima, "Single mode extraction from multiple modes of lamb wave and its application to defect detection," JSME Int. J. Series A 46, 620-626 (2003).

${ }^{60} \mathrm{~K}$. Xu, D. Ta, and W. Wang, "Multiridge-based analysis for separating individual modes from multimodal guided wave signals in long bones," IEEE Trans. Ultrason. Ferroelectr. Freq. Control 57, 2480-2490 (2010).

${ }^{61} \mathrm{~F}$. Gao, L. Zeng, J. Lin, and Z. Luo, "Mode separation in frequency-wavenumber domain through compressed sensing of far-field Lamb waves," Meas. Sci. Technol. 28, 075004 (2017).

${ }^{62}$ M. Ratassepp, A. Klauson, F. Chati, F. Léon, D. Décultot, G. Maze, and M. Fritzsche, "Application of orthogonality-relation for the separation of Lamb modes at a plate edge: Numerical and experimental predictions," Ultrasonics 57, 90-95 (2015).

${ }^{63} \mathrm{X}$. Song, D. Ta, and W. Wang, "Analysis of superimposed ultrasonic guided waves in long bones by the joint approximate diagonalization of eigen-matrices algorithm," Ultrasound Med. Biol. 37, 1704-1713 (2011). 